\title{
A FUNDAMENTAL SOLUTION OF THE PARABOLIC EQUATION ON HILBERT SPACE. II: THE SEMIGROUP PROPERTY
}

\author{
BY \\ M. ANN PIECH
}

\begin{abstract}
The existence of a family of solution operators $\left\{q_{t}: t>0\right\}$ corresponding to a fundamental solution of a second order infinite-dimensional differential equation of the form $\partial u / \partial t=L u$ was previously established by the author. In the present paper, it is established that these operators are nonnegative, and satisfy the condition $q_{s} q_{t}=q_{s+t}$
\end{abstract}

I. Introduction. This paper continues the study initiated in [12] of second order parabolic equations, with variable coefficients, on Hilbert space. In [12] we established a fundamental solution for the equation $\partial u / \partial t=L u$, where $L$ is a second order differential operator satisfying certain regularity hypotheses. This fundamental solution is given by a family of finite signed Borel measures $\left\{q_{t}(x, d y): t>0, x \in B\right\}$ on a Banach space $B$ ( $B$ will be defined later) or, equivalently, by a family of operators $\left\{q_{t}: t>0\right\}$ on the space of bounded Lip-1 functions on $B$. These operators were defined via infinite series, which made it difficult to determine either their nonnegativity or whether they satisfy a semigroup property $\left(q_{s} q_{t}=q_{t+s}\right.$ for all $s, t>0)$.

The technique developed in this paper for establishing both nonnegativity and the semigroup property is that of "semifinite" approximation. Basically, the differential operator $L$ is approximated by a differential operator $L^{K}$ acting in a finitedimensional subspace $K$ of our Hilbert space $H$ plus the Laplacian $\Delta$ acting in $K^{\perp}$. Nonnegativity and the semigroup property are known for the fundamental solutions of $\partial u / \partial t=L^{K} u$ and $\partial u / \partial t=\Delta u$. Combining these fundamental solutions and passing to the limit as $K \rightarrow H$ in some suitable fashion, we obtain the desired properties for $\left\{q_{t}\right\}$.

II. Preliminaries. Most of the basic definitions and ideas necessary to the following work can be found in Gross [8], [9] and in the preliminaries of [12]. The notation is that of [12] to the extent to which that is possible.

Let $H$ denote a real separable Hilbert space with norm $|\cdot|$ and inner product

Received by the editors November 10, 1969.

AMS Subject Classifications. Primary 2846, 4615, 4750; Secondary 3562.

Key Words and Phrases. Hilbert space, abstract Wiener space, Wiener measure, parabolic equation, fundamental solution, semigroups of operators.

Copyright (C) 1970, American Mathematical Society 
( , ). Gauss measure on $H$ with variance parameter $t$ is denoted by $\nu_{t}$, and is defined for a cylinder set $S \subset H$ by

$$
\nu_{t}(S) \equiv(2 \pi t)^{-n / 2} \int_{E} \exp \left[-|x|^{2} / 2 t\right] d x
$$

where $S=P^{-1}(E), P$ being an $n$-dimensional projection on $H$ and $E$ a Borel set in the range of $P$.

Let $\|\cdot\|$ denote a particular measurable norm on $H$, and let $B$ be the completion of $H$ with respect to $\|\cdot\|$. The triple $(H, B, i)$, where $i$ is the natural injection of $H$ into $B$, is called an abstract Wiener space. Gauss measure $\nu_{t}$ on $H$ induces a Borel measure $p_{t}$ on $B$ which is such that

$$
p_{t}\left\{x \in B /\left(\left\langle y_{1}, x\right\rangle, \ldots,\left\langle y_{n}, x\right\rangle\right) \in E\right\}=\nu_{t}\left\{x \in H /\left(\left\langle y_{1}, x\right\rangle, \ldots,\left\langle y_{n}, x\right\rangle\right) \in E\right\}
$$

for all finite subsets $y_{1}, \ldots, y_{n}$ of $B^{*}$ and Borel sets $E \subset R^{n}$. (Here we identify $B^{*}$ with a subset of $H^{*}$.) $p_{t}$ is called Wiener measure on $B$ with variance parameter $t$.

Certain functions $f$ defined on $H$ determine measurable functions on $B$. The manner in which this takes place is described in Gross [7] for tame functions on $H$ and for functions which are uniformly continuous near zero in $H_{m}$ (u.c.n. 0 in $H_{m}$ ), where $H_{m}$ denotes $H$ with the topology determined by the measurable seminorms. The measurable function on $B$ determined in this fashion by $f$ is denoted by $f$. We will generally omit the tilde whenever it is obvious that we are working on $B$-e.g. $\int_{B} f(y) p_{t}(d y)$. In this paper we assume that $\|y\|$ in is $L^{p}\left(p_{t}(d y)\right)$ for all $1 \leqq p<\infty$ and for all $t>0$.

Let $W$ be any Banach space. If $f$ is a $W$-valued function defined in a neighborhood of a point $x$ of $B$, we will write $D f(x)$ for the Fréchet derivative of $f$ at $x$, and will call $f B$-differentiable at $x$ if $D f(x)$ exists. We may also regard $f$ as a function $g$ defined on a neighborhood of the origin of $H$ by restricting $f$ to the coset $x+H$ of $B$ and defining $g(h) \equiv f(x+h)$. The Fréchet derivative of $g$ at 0 is denoted by $f^{\prime}(x)$, and we say that $f$ is $H$-differentiable at $x$ if $f^{\prime}(x)$ exists. We write $\|D f(x)\|$ and $\left|f^{\prime}(x)\right|$ for the $L(B, W)$ and $L(H, W)$ norms respectively.

We will now briefly sketch the results of [12]. Let $A(x) \equiv I-B(x)$, where $B(\cdot)$ is a map from $B$ to the space of symmetric trace class operators on $H$. For a realvalued measurable function $f(x, t)$ on $B \times(0, \infty)$ we define

$$
L_{x, t} f(x, t) \equiv \operatorname{trace}\left[A(x) f^{\prime \prime}(x, t)\right]-(\partial / \partial t) f(x, t) \quad(0<t<\infty)
$$

whenever the right-hand side exists-that is, whenever $(\partial / \partial t) f(x, t)$ and $f^{\prime \prime}(x, t)$ exist and $\left[A(x) f^{\prime \prime}(x, t)\right]$ is trace class. When there is no danger of confusion, we will omit the subscripts on $L$. We assume that $B(x)$ satisfies the following hypotheses:

(a-1) $x \rightarrow B(x)$ is a bounded Lip-1 function from $B$ to the space of symmetric trace class operators on $H$, with the trace class norm.

(a-2) There exists $\varepsilon_{0}>0$ such that $B(x) \leqq\left(1-\varepsilon_{0}\right) I$ for all $x \in B$.

(a-3) There exists a symmetric Hilbert-Schmidt class operator $E$ on $H$ and a 
family of operators $B_{0}(x) \in L(H, H)$ such that for all $x \in B, B(x)=E B_{0}(x) E$ and $\left|B_{0}(x)\right| \leqq 1$.

(a-4) $D^{2} B_{0}(x)$ exists and is a Lip-1 function from $B$ to $L(B \rightarrow L(B \rightarrow L(H, H)))$.

(a-5) $\left\|D B_{0}(x)\right\|,\left\|D^{2} B_{0}(x)\right\|$ are uniformly bounded for all $x \in B$.

(a-6) There exists a constant $c$ such that for any orthonormal basis $\left\{e_{i}: i=1\right.$, $2, \ldots\}$ of $H$ we have $\sum_{i=1}^{\infty}\left|D B_{0}(x) e_{i}\right|^{2}<c$, independently of $x \in B$.

REMARKs. (1) Without loss of generality we may assume that $\varepsilon_{0}<1$.

(2) (a-6) is always satisfied if $B$ is the completion of $H$ with respect to a measurable norm of the form $\|y\|=|S y|$ for all $y \in H$, where $S$ is a Hilbert-Schmidt operator on $H$. For then $\sum_{i=1}^{\infty}\left|D B_{0}(x) e_{i}\right|^{2}$ is dominated for all $x$ by a constant times $\sum_{i=1}^{\infty}\left\|e_{i}\right\|^{2}$ (by (a-5)), and we have

$$
\sum_{i=1}^{\infty}\left\|e_{i}\right\|^{2}=\sum_{i=1}^{\infty}\left|S e_{i}\right|^{2}=(\text { the Hilbert-Schmidt norm of } S)^{2} .
$$

(3) The argument given on p. 107 of [12] for the operator denoted there as $\left(C^{\prime}(x)(\cdot)(\cdot), y\right)$ to be of trace class is incorrect. (a-6) is a sufficient, but by no means necessary, condition for this operator to be of trace class. We will show this in detail in the proof of $(c-11)$ of $\S \mathrm{V}$.

Under the preceding hypotheses on $B(x)$ (and therefore on $A(x)$ ), there exists a family of finite real-valued signed Borel measures $\left\{q_{t}(x, d y): 0<t<\infty, x \in B\right\}$ on $B$ such that if $q_{t} f(x) \equiv \int_{B} f(y) q_{t}(x, d y)$ then for each bounded Lip-1 function $f$ from $B$ to the reals we have $L_{x, t} q_{t} f(x)=0$ (for all $0<t<\infty, x \in B$ ) and $\lim _{t \rightarrow 0} q_{t} f(x)=f(x)$ uniformly in $x$.

III. The semifinite approximation. Consider a finite-dimensional subspace $K$ of $B$ of the following form: Let $y_{1}, \ldots, y_{n}$ be a set of orthonormal vectors in $H^{*}$ which also lie in $B^{*}$. Let $K \equiv \operatorname{span}\left(y_{1}, \ldots, y_{n}\right)$. Then if $P$ is the continuous extension to $B$ of the orthogonal projection of $H$ onto $K$, we have

$$
P x=\sum_{i=1}^{n}\left\langle y_{i}, x\right\rangle y_{i}, \quad(x \in B)
$$

and $P$ is a projection on $B$.

In order to carry out our approximations, we must make three further assumptions. They are as follows:

(a-7) There exists a sequence $\left\{P_{n}\right\}$ of commuting finite-dimensional projections on $B$, of the above form, such that $\left\{P_{n}\right\}$ converges strongly to the identity operator on $B$.

(a-8) $E$ (see (a-3)) commutes with each $P_{n}$.

(a-9) For each $x \in B$ and $P_{n}$ from (a-7), there exists a constant $c_{x, P_{n}}$ such that

$$
\sum_{i=1}^{\infty}\left|\left[D B_{0}\left(P_{n} x\right)-D B_{0}(x)\right] e_{i}\right|^{2}<c_{x, P_{n}}
$$

for every orthonormal basis $\left\{e_{i}\right\}$ of $H$, and $c_{x, P_{n}} \rightarrow 0$ as $n \rightarrow \infty$. 
Remarks. (1) By considering pairwise least upper bounds, we may assume, without loss of generality, that $P_{n+1} \supseteq P_{n}$.

(2) All projections which occur in this paper will be selected from this sequence, and the subscripts will be omitted-so that $P$ will denote an arbitrary member of this sequence, corresponding to projection on the finite-dimensional subspace $K$ (where we may consider $K$ as a subspace of $B^{*}$ or of $B$ or of $H$ ).

(3) (a-7) is valid in the case of Wiener space. Let $B$ be the space of real continuous functions on $[0,1]$ which vanish at zero and let $H$ be that subset of $B$ consisting of the absolutely continuous functions which have square integrable first derivatives. The inner product on $H$ is given by

$$
(x, y) \equiv \int_{0}^{1} x^{\prime}(t) y^{\prime}(t) d t
$$

where ' denotes the first derivative with respect to $t . B$ is the completion of $H$ with respect to the sup norm $\left(\|\cdot\|_{\infty}\right)$. We first construct a basis for $H^{*}$ consisting of elements of $B^{*}$. For this purpose we use the Haar functions $\left\{\chi_{n}(t)\right\}$, which are defined by

$$
\begin{aligned}
\chi_{1}(t) & \equiv 1 & & t \in[0,1], \\
\chi_{2^{n}+k}(t) & \equiv \sqrt{ } 2^{n} & & t \in\left[(k-1) / 2^{n},\left(k-\frac{1}{2}\right) / 2^{n}\right), \\
& \equiv-\sqrt{ } 2^{n} & & t \in\left(\left(k-\frac{1}{2}\right) / 2^{n}, k / 2^{n}\right], \\
& \equiv 0 & & \text { otherwise in }[0,1],
\end{aligned}
$$

for $n=0,1,2, \ldots, k=1,2, \ldots, 2^{n}$. It is well known that $\left\{\chi_{n}(t)\right\}$ forms a complete orthonormal set in $L^{2}[0,1]$ (using Lebesgue measure) ([15, p. 338]). Let $y_{n}(t) \equiv$ $\int_{0}^{t} \chi_{n}(s) d s$. It is obvious that $\left\{y_{n}\right\}$ forms a complete orthonormal set in $H^{*}$. For $x \in H$, we have the formulas

$$
\begin{aligned}
\left\langle y_{1}, x\right\rangle & =\int_{0}^{1} x^{\prime}(t) d t=x(1), \\
\left\langle y_{2^{n}+k}, x\right\rangle & =\int_{0}^{1} x_{2^{n}+k}(t) x^{\prime}(t) d t \\
& =\int_{(k-1) / 2^{n}}^{(k-1 / 2) / 2^{n}} \sqrt{ } 2^{n} x^{\prime}(t) d t-\int_{(k-1 / 2) / 2^{n}}^{k / 2^{n}} \sqrt{ } 2^{n} x^{\prime}(t) d t \\
& =\sqrt{ } 2^{n}\left[2 x\left(\frac{k-\frac{1}{2}}{2^{n}}\right)-x\left(\frac{k-1}{2^{n}}\right)-x\left(\frac{k}{2^{n}}\right)\right] .
\end{aligned}
$$

We may now use these formulas to define $\left\langle y_{n}, x\right\rangle$ for all $x \in B . y_{n} \in B^{*}$ since $\left|\left\langle y_{n}, x\right\rangle\right| \leqq 4 \sqrt{ } 2^{n}\|x\|_{\infty}$. Moreover, for each $x \in B$,

$$
x(t)=\sum_{n=1}^{\infty}\left\langle y_{n}, x\right\rangle y_{n}(t),
$$


the convergence being uniform in $t$ [1, Theorem 3]. If we now define

$$
P_{n} x \equiv \sum_{i=1}^{n}\left\langle y_{i}, x\right\rangle y_{i}
$$

we have a sequence satisfying (a-7).

(4) If $B$ is itself a Hilbert space, with inner product [, ], then $x, y \rightarrow[x, y]$ is a bilinear functional on $H$. Since $|[x, y]| \leqq\|x\| \cdot\|y\| \leqq c|x| \cdot|y|$ for some constant $c$, this bilinear form is bounded. Thus there exists a positive definite operator $N$ on $H$ such that $(N x, y)=[x, y] \cdot \sqrt{ } N$ is completely continuous, since $|\sqrt{ } N x|=[x, x]^{1 / 2}=$ $\|x\|$ and the injection mapping from $H \rightarrow B$ is completely continuous [8]. Let $\left\{y_{i}\right\}$ be an orthonormal basis for $H$ consisting of eigenvectors of $\sqrt{ } N$, with $\left\{\lambda_{i}\right\}$ the corresponding sequence of eigenvalues. Each $\lambda_{i}>0$ and $\lambda_{i} \rightarrow 0 .\left\{\lambda_{i}^{-1} y_{i}\right\}$ forms an orthonormal basis for $B$. Considering $y_{i}$ to be in $H^{*}$, we have

$$
\left\langle y_{i}, x\right\rangle=\left(y_{i}, x\right)=\left(N\left(\lambda_{i}^{-2} y_{i}\right), x\right)=\lambda_{i}^{-1}\left[\lambda_{i}^{-1} y_{i}, x\right] \text { for all } x \in H .
$$

This formula makes sense for all $x \in B$, and so defines the unique extension of $y_{i}$ to an element of $B^{*}$. Now for $x \in B$,

$$
\sum_{i=1}^{n}\left\langle y_{i}, x\right\rangle y_{i}=\sum_{i=1}^{n}\left[\lambda_{i}^{-1} y_{i}, x\right] \lambda_{i}^{-1} y_{i} \rightarrow x
$$

in the $B$-norm. Defining $P_{n} x \equiv \sum_{i=1}^{n}\left\langle y_{i}, x\right\rangle y_{i}$, we see that (a-7) is thus satisfied whenever $B$ is a Hilbert space.

(5) (a-9) is satisfied whenever $B$ is of the form defined in Remark (2) following (a-6), since in this case (a-5) gives

$$
\left|\left[D B_{0}(P x)-D B_{0}(x)\right] y\right| \leqq \text { constant } \cdot\|P x-x\| \cdot\|y\|
$$

for each $y \in H$.

Define

$$
\begin{aligned}
A^{K}(x) & \equiv I-P B(P x) P \quad(x \in B) \\
& =(I-P)+(P-P B(P x) P) \\
& \equiv Q+A^{P}(x), \quad \text { say, where } P H=K \text { as stated before. }
\end{aligned}
$$

Considering $K \subset B^{*}$, denote by $K^{\perp}$ the annihilator of $K$ in $B$. Then if $\nu_{t}^{\prime}$ denotes Gauss measure on $K$, and $p_{t}^{\prime \prime}$ denotes Wiener measure on $K^{\perp}$, we have [9, p. 131, Remark 2.2] $p_{t}=\nu_{t}^{\prime} \times p_{t}^{\prime \prime}$ and thus $p_{t}(x, d y)=v_{t}^{\prime}\left(x^{\prime}, d y^{\prime}\right) \times p_{t}^{\prime \prime}\left(x^{\prime \prime}, d y^{\prime \prime}\right)$ (for all $x \in B$, $t>0)$, where $x=x^{\prime}+x^{\prime \prime}, y=y^{\prime}+y^{\prime \prime}, x^{\prime}$ and $y^{\prime} \in K, x^{\prime \prime}$ and $y^{\prime \prime} \in K^{\perp}$.

Notation. If $\omega_{t}(x, d y)$ is a finite real-valued signed Borel measure on a space $W$, then for a Borel function $f$ on $W$ we define

$$
\left(\omega_{t} f\right)(x) \equiv \int_{w} f(y) \omega_{t}(x, d y)
$$


when this integral exists. (A finite Borel measure is one such that $\left|\omega_{t}(x, E)\right|<\infty$ for all Borel sets $E \subset W$. For a real-valued measure, finiteness is equivalent to bounded variation, by the Hahn Decomposition Theorem.)

If $f(x, t)$ is a real-valued Borel measurable function on $K \times(0, \infty)$, define

$$
L_{x, t}^{P} f(x, t) \equiv \operatorname{trace}_{K}\left[A^{P}(x) f_{x x}(x, t)\right]-\partial / \partial t f(x, t)
$$

for all $t>0, x \in K$, whenever the right-hand side exists. Here $f_{x x}(x, t)$ denotes the second Fréchet derivative of $f$ with respect to $x . L_{x, t}^{P}$ is a parabolic operator in $K$. By the finite-dimensional theory [2], [3], [4], [5], [10], [11] there exists a family of functions $\left\{q^{\prime}(t, x, y)\right\}$, where $t>0, x$ and $y \in K$, which satisfies

(i) $q^{\prime}(t, x, y)$ is jointly continuous in $t, x$, and $y$;

(ii) $q_{x x}^{\prime}(t, x, y)$ and $\partial / \partial t q^{\prime}(t, x, y)$ exist and $L_{x, t}^{P} q^{\prime}(t, x, y)=0$ on $(0, \infty) \times K \times K$;

(iii) if $f(x)$ is bounded and continuous on $K$, then

$$
\lim _{t \backslash 0} \int_{K} f(y) q^{\prime}(t, x, y) d y=f(x) \quad(x \in K)
$$

where the convergence is uniform on compact subsets of $K$;

(iv) for any $\varepsilon$ and $t_{0}>0, q^{\prime}(t, x, y)$ is bounded on the set

$$
\left\{t+|x-y| \geqq \varepsilon, 0<t \leqq t_{0}\right\} .
$$

Moreover, $q^{\prime}(t, x, y)$ is unique among functions which satisfy (i)-(iv).

It is not difficult [3], [4], [5], [11] to show that the construction of a fundamental solution for the equation $L_{x, t}^{P} f=0$ described in [12] (in this case $K$ is the Hilbert space under consideration) produces a family of finite signed Borel measures $\left\{q_{t}^{\prime}(x, d y)\right\}$ on $K$ which are of the form $q_{t}^{\prime}(x, d y)=q^{\prime}(t, x, y) d y$ where $q^{\prime}(t, x, y)$ satisfies properties (i)-(iv). (In $q^{\prime}(t, x, y) d y$, the $d y$ refers to Lebesgue measure on $K$.) It now follows from Dynkin [4, Chapter V] that the family $\left\{q_{t}^{\prime}: t>0\right\}$ forms a contraction semigroup of positive operators acting on the space $\mathscr{B}(K)$ of bounded Borel functions on $K$. Moreover,

$$
q^{\prime}(t, x, y)>0 \quad(t>0, x \text { and } y \in K),
$$

and

$$
\int_{K} q^{\prime}(t, x, y) d y=1 \quad(t>0, x \in K) .
$$

(The last property is found in [11].)

We define a family of finite Borel measures $\left\{q_{t}^{K}(x, d y)\right\}$ on $B$ by

$$
q_{t}^{K}(x, d y) \equiv q_{t}^{\prime}\left(x^{\prime}, d y^{\prime}\right) \times p_{2 t}^{\prime \prime}\left(x^{\prime \prime}, d y^{\prime \prime}\right) \quad(t>0, x \in B) .
$$

REMARK. The family $\left\{p_{t}^{\prime \prime}(x, d y): t>0, x \in K^{\perp}\right\}$ is a fundamental solution of the heat equation $\partial / \partial t f(x, t)=\frac{1}{2} \operatorname{trace}_{K^{\perp}}\left[f^{\prime \prime}(x, t)\right]$ in $K^{\perp}$ (see Gross [4, Theorem 3]). A straightforward change of variables shows that the factor of $\frac{1}{2}$ in the heat equation may be removed by considering the family $\left\{p_{2 t}^{\prime \prime}(x, d y)\right\}$. 
Proposition 1. $\left\{q_{t}^{K}: t>0\right\}$ is a contraction semigroup of positive operators acting in the space $\mathscr{B}(B)$ of bounded Borel functions on $B$ with the sup norm $\|\cdot\|_{\infty}$.

Proof. If $E$ is a Borel set in $B$, of the form $E=E^{\prime} \times E^{\prime \prime}$ where $E^{\prime} \subset K$ and $E^{\prime \prime} \subset K^{\perp}$, then

$$
\left(q_{t}^{K} \chi_{E}\right)(x)=\left\{\left(q_{t}^{\prime} \chi_{E}^{\prime}\right)\left(x^{\prime}\right)\right\}\left\{\left(p_{2 t}^{\prime \prime} \chi_{E^{\prime \prime}}\right)\left(x^{\prime \prime}\right)\right\},
$$

and each function on the right-hand side is a Borel function of $x$. The set $\mathscr{S}$ of all Borel sets $E$ such that $q_{t}^{K} \chi_{E}$ is measurable is clearly closed under finite disjoint unions, and so contains the field generated by sets $E$ of the above form $E^{\prime} \times E^{\prime \prime}$. Since $\mathscr{S}$ is closed under monotone limits, it follows that $\mathscr{S}$ coincides with the $\sigma$-field of Borel sets. The set of all $f$ for which $q_{t}^{K} f$ is a Borel function contains the characteristic functions of the Borel sets and is closed under bounded monotone limits, and thus contains all $f \in \mathscr{B}(B)$. Since $q_{t}^{K}(x, d y)$ is a probability measure for each $x \in B$ and $t>0$, we have $\left\|q_{t}^{K} f\right\|_{\infty} \leqq\|f\|_{\infty}$.

To prove the semigroup property, we note that for $E$ of the above form

$$
\begin{aligned}
\int_{B ;(z)} \int_{B ;(y)} \chi_{E}(y) \cdot q_{t}^{K}(z, d y) & \cdot q_{s}^{K}(x, d z) \\
& =\int_{B} q_{t}^{\prime}\left(z^{\prime}, E^{\prime}\right) \cdot p_{2 t}^{\prime \prime}\left(z^{\prime \prime}, E^{\prime \prime}\right) \cdot q_{s}^{K}(x, d z) \\
& =\left\{\int_{K} q_{t}^{\prime}\left(z^{\prime}, E^{\prime}\right) \cdot q_{s}^{\prime}\left(x^{\prime}, d z^{\prime}\right)\right\} \cdot\left\{\int_{K^{\perp}} p_{2 t}^{\prime \prime}\left(z^{\prime \prime}, E^{\prime \prime}\right) \cdot p_{2 s}^{\prime \prime}\left(x^{\prime \prime}, d z^{\prime \prime}\right)\right\} \\
& =q_{s+t}^{\prime}\left(x^{\prime}, E^{\prime}\right) \cdot p_{2(s+t)}^{\prime \prime}\left(x^{\prime \prime}, E^{\prime \prime}\right) \\
& =q_{s+t}^{K}(x, E) \\
& =\int_{B} \chi_{E}(y) q_{s+t}^{K}(x, d y) .
\end{aligned}
$$

The set of all $f$ for which

$$
\int_{B ;(z)} \int_{B ;(y)} f(y) \cdot q_{t}^{K}(z, d y) \cdot q_{s}^{K}(x, d z)=\int_{B} f(y) q_{s+t}^{K}(x, d y)
$$

is closed under finite linear combinations and under bounded monotone limits, and so by the preceding argument contains all $f \in \mathscr{B}(B)$. Thus we have established that $q_{s}^{K}\left(q_{t}^{K}\right) f=q_{s+t}^{K} f$ for all $f \in \mathscr{B}(B)$.

We next establish some notation and define some properties of measures. For any metric space $W$ with metric $d$, let $\mathscr{B}(W)$ denote the space of all bounded realvalued Borel functions with the sup norm $\|\cdot\|_{\infty}$ and let $\mathscr{A}(W)$ be the space of all real bounded Lip-1 functions on $W$ with norm $\|\cdot\|_{1}$ defined by

$$
\|f\|_{1} \equiv\|f\|_{\infty}+\inf \{c:|f(x)-f(y)| \leqq c \cdot d(x, y) \text { for all } x, y \in W\} .
$$

For a family $\left\{\omega_{t}(x, d y): x \in W, t>0\right\}$ of finite real-valued signed Borel measures on $W$, we define the following properties: 
(b-1) There exists a constant $c$, independent of $t$ and $x$, such that

$$
\int_{W}\left|\omega_{t}\right|(x, d y) \leqq c t^{-1 / 2} \text { for all } x \in W, t>0 .
$$

(Here $\left|\omega_{t}\right|(x, E)$ denotes the variation of $\omega_{t}(x, d y)$ over $E$ for each Borel set $E$ in $W$.)

(b-1(a)) Given $0<t_{0}<\infty$, there exists a constant $c_{t_{0}}$, independent of $t$ and $x$, such that

$$
\int_{W}\left|\omega_{t}\right|(x, d y) \leqq c_{t_{0}} t^{-1 / 2} \quad \text { for all } x \in W, 0<t \leqq t_{0} .
$$

(b-2) The map $f \rightarrow \omega_{t} f$ defined by $\left(\omega_{t} f\right)(x) \equiv \int_{w} f(y) \omega_{t}(x, d y)$ is a bounded linear operator on $\mathscr{B}(W)$ for each $t>0$.

(b-3) (b-1) holds and $f \rightarrow \omega_{t} f$ is a bounded linear operator on $\mathscr{A}(W)$, with

$$
\left\|\omega_{t} f\right\|_{1} \leqq c t^{-1 / 2}\|f\|_{1} \text { for all } t>0
$$

where $c$ is given by (b-1).

(b-3(a)) (b-1(a)) holds and $f \rightarrow \omega_{t} f$ is a bounded linear operator on $\mathscr{A}(W)$, with

$$
\left\|\omega_{t} f\right\|_{1} \leqq c_{t_{0}} t^{-1 / 2}\|f\|_{1} \quad \text { for all } 0<t \leqq t_{0},
$$

where $c_{t_{0}}$ is given by (b-1(a)).

(b-4) Given $0<\delta \leqq t_{0}<\infty$, there exists a constant $c_{\delta, t_{0}}$, independent of $f$ and $x$, such that for $\delta \leqq t_{1}, t_{2} \leqq t_{0}$ we have

$$
\left|\left(\omega_{t_{1}} f\right)(x)-\left(\omega_{t_{2}} f\right)(x)\right| \leqq c_{\delta, t_{0}}\left|t_{1}-t_{2}\right| \cdot\|f\|_{1}
$$

for all $f \in \mathscr{A}(W)$ and $x \in W$.

It is a consequence of [13, Propositions 4 and 5] that if the family $\left\{\omega_{t}(x, d y)\right\}$ satisfies (b-3) or (b-3(a)), then it must satisfy (b-2).

Define the family $\left\{m_{t}^{K}(x, d y): t>0, x \in B\right\}$ of finite Borel measures on $B$ by

$$
m_{t}^{K}(x, d y) \equiv \exp \left[-\left(C^{K}(x)(x-y), x-y\right) / 4 t\right]^{\sim} p_{2 t}(x, d y)
$$

where $C^{K}(x) \equiv\left[A^{K}(x)\right]^{-1}-I=[I-P B(P x) P]^{-1} P B(P x) P$. On $K^{\perp}, C^{K}(x)$ acts as the zero operator. $K$ is invariant under $C^{K}(x)$, and, if we define

$$
C^{P}(x) \equiv[P-P B(P x) P]^{-1} P B(P x) P \in L(K, K)
$$

then $C^{K}(x)=C^{P}(x)$ on $K$. Thus we can write

$$
\begin{aligned}
m_{t}^{K}(x, d y) & =\exp \left[-\left(C^{P}\left(x^{\prime}\right)\left(x^{\prime}-y^{\prime}\right), x^{\prime}-y^{\prime}\right) / 4 t\right] \nu_{2 t}^{\prime}\left(x^{\prime}, d y^{\prime}\right) \times p_{2 t}^{\prime \prime}\left(x^{\prime \prime}, d y^{\prime \prime}\right) \\
& \equiv m_{t}^{P}\left(x^{\prime}, d y^{\prime}\right) \times p_{2 t}^{\prime \prime}\left(x^{\prime \prime}, d y^{\prime \prime}\right)
\end{aligned}
$$

where $x=x^{\prime}+x^{\prime \prime}, y=y^{\prime}+y^{\prime \prime}, x^{\prime}$ and $y^{\prime} \in K, x^{\prime \prime}$ and $y^{\prime \prime} \in K^{\perp}$. We may also define

$$
\begin{aligned}
\hat{m}_{t}^{K}(x, d y) & \equiv\left[\operatorname{det} A^{K}(y)\right]^{-1 / 2} m_{t}^{K}(x, d y) \\
& =\left[\operatorname{det} A^{P}(P y)\right]^{-1 / 2} m_{t}^{K}(x, d y) \\
& =\left[\operatorname{det} A^{P}\left(y^{\prime}\right)\right]^{-1 / 2} m_{t}^{P}\left(x^{\prime}, d y^{\prime}\right) \times p_{2 t}^{\prime \prime}\left(x^{\prime \prime}, d y^{\prime \prime}\right) \\
& \equiv \hat{m}_{t}^{P}\left(x^{\prime}, d y^{\prime}\right) \times p_{2 t}^{\prime \prime}\left(x^{\prime \prime}, d y^{\prime \prime}\right) .
\end{aligned}
$$


All the measures which we have defined are finite Borel measures on the appropriate spaces.

We may now "apply" $L_{x, t}^{K}$ to $\hat{m}_{t}^{K}(x, d y)$ as in [12, Proposition 2], obtaining a family $\left\{M_{t}^{K}(x, d y)\right\}$ of Borel measures on $B$ satisfying (b-1)-(b-3). We observe from equation (19) of [12] that we may write

$$
M_{t}^{K}(x, d y)=M_{t}^{P}\left(x^{\prime}, d y^{\prime}\right) \times p_{2 t}^{\prime \prime}\left(x^{\prime \prime}, d y^{\prime \prime}\right)
$$

where $M_{t}^{P}\left(x^{\prime}, d y^{\prime}\right)$ acts in $K$ and is given by

$$
\begin{aligned}
& M_{t}^{P}(x, d y) \equiv\left[\operatorname{det} A^{P}(y)\right]^{-1 / 2}\left\{\operatorname{trace}_{K}\left[A^{P}(x)\right][\right.-4(t)^{-1}\left(C^{P^{\prime \prime}}(x)(\cdot)(\cdot)(x-y), x-y\right) \\
&-t^{-1}\left(C^{P^{\prime}}(x)(\cdot)(\cdot), x-y\right) \\
&+\left(16 t^{2}\right)^{-1}\left(C^{P^{\prime}}(x)(\cdot)(x-y), x-y\right) \\
&\left.\otimes\left(C^{P^{\prime}}(x)(\cdot)(x-y), x-y\right)\right]\left.+(4 t)^{-1}\left(C^{P^{\prime}}(x)(x-y)(x-y), x-y\right)\right\} \\
&4) \quad \exp \left[-\left(C^{P}(x)(x-y), x-y\right) / 4 t\right] \cdot v_{2 t}(x, d y)
\end{aligned}
$$

for all $t>0, x$ and $y \in K$. The symmetric operator $T \in L(K, K)$ which is denoted by $\left(C^{P^{\prime}}(x)(\cdot)(\cdot), x-y\right)$ is defined by

$$
\left(T k_{1}, k_{2}\right) \equiv \frac{1}{2}\left[\left(C^{P^{\prime}}(x) k_{1} k_{2}, x-y\right)+\left(C^{P^{\prime}}(x) k_{2} k_{1}, x-y\right)\right] \text { for all } k_{1}, k_{2} \in K .
$$

If we replace $P$ by $I$ and $\nu_{2 t}$ by $p_{2 t}$ in (4) $\left(A^{I}(y) \equiv A(y), C^{I}(x) \equiv C(x)\right)$, then we obtain the measures $\left\{M_{t}(x, d y)\right\}$ of [12]. We may also replace $P$ by $I$ in (2) and (3), obtaining $\left\{m_{t}(x, d y)\right\}$ and $\left\{\hat{m}_{t}(x, d y)\right\}$.

Proposition 2. The family $\left\{q_{t}^{K}(x, d y): t>0, x \in B\right\}$ coincides with the fundamental solution of

$$
L_{x, t}^{K} f(x, t) \equiv \operatorname{trace}_{H}\left[A^{K}(x) f^{\prime \prime}(x, t)\right]-\partial / \partial t f(x, t)=0
$$

obtained by the method of [12].

LEMMA 2.1. If $\left\{M_{t}: t>0\right\}$ is any family of operators on $\mathscr{B}(B)$ which satisfies an inequality of the form $\left\|M_{t} f\right\|_{\infty} \leqq Q t^{-1 / 2}\|f\|_{\infty}$ for some constant $Q$ independent of $t>0$ and of $f \in \mathscr{B}(B)$, then any family $\left\{r_{t}(x, d y): t>0, x \in B\right\}$ of real-valued signed Borel measures on $B$ which satisfies

$$
r_{t} f(x)=M_{t} f(x)+\int_{0}^{t} M_{t-u}\left[r_{u} f\right](x) d u
$$

for all $f \in \mathscr{B}(B)$ and property (b-1(a)) is unique.

Proof. Assume that $\left\{\boldsymbol{r}_{t}(x, d y)\right\}$ and $\left\{\bar{r}_{t}(x, d y)\right\}$ each satisfy (6) and (b-1(a)). Without loss of generality we may assume that the constants $c_{t_{0}}$ of $(b-1(a))$ are the same for both families. Then for $f \in \mathscr{B}(B)$

$$
r_{t} f(x)-\bar{r}_{t} f(x)=\int_{0}^{t} M_{t-u}\left[r_{u} f-\bar{r}_{u} f\right](x) d u .
$$


Now

$$
\left\|M_{t-u}\left[r_{u} f-\bar{r}_{u} f\right]\right\|_{\infty} \leqq Q(t-u)^{-1 / 2}\left\|r_{u} f-\bar{r}_{u} f\right\|_{\infty} \quad \text { for all } 0<u<t<\infty .
$$

Thus for all $0<t \leqq t_{0}$ we have

$$
\begin{aligned}
\left\|r_{t} f-\bar{r}_{t} f\right\|_{\infty} & \leqq Q \int_{0}^{t}(t-u)^{-1 / 2}\left\|r_{u} f-\bar{r}_{u} f\right\|_{\infty} d u \\
& \leqq 2 c_{t_{0}}\|f\|_{\infty} Q \int_{0}^{t}(t-u)^{-1 / 2} u^{-1 / 2} d u \\
& =2 c_{t_{0}}\|f\|_{\infty} Q \pi^{2 / 2} t^{2 / 2-1}(\Gamma(2 / 2))^{-1}
\end{aligned}
$$

Iterating, we get

$$
\begin{aligned}
\left\|r_{t} f-\bar{r}_{t} f\right\|_{\infty} & \leqq 2 c_{t_{0}}\|f\|_{\infty} Q^{2} \pi^{2 / 2}(\Gamma(2 / 2))^{-1} \int_{0}^{t}(t-u)^{-1 / 2} u^{2 / 2-1} d u \\
& =2 c_{t_{0}}\|f\|_{\infty} Q^{2} \pi^{3 / 2} t^{3 / 2-1}(\Gamma(3 / 2))^{-1}
\end{aligned}
$$

and eventually obtain

$$
\left\|r_{t} f-\bar{r}_{t} f\right\|_{\infty} \leqq 2 c_{t_{0}}\|f\|_{\infty} Q^{n-1} \pi^{n / 2} t^{n / 2-1}(\Gamma(n / 2))^{-1}
$$

for each $n=2,3, \ldots$ But $Q^{n-1} \pi^{n / 2} t^{n / 2-1}(\Gamma(n / 2))^{-1}$ goes to zero as $n \rightarrow \infty$, for each $t>0$. Therefore $\left\|r_{t} f-\bar{r}_{t} f\right\|_{\infty}=0$ for all $t>0$, and so $r_{t} f=\bar{r}_{t} f$ for all $f \in \mathscr{B}(B)$ and in particular $r_{t}(x, E)=\bar{r}_{t}(x, E)$ for all Borel sets $E \subset B$.

Proof of Proposition 2. From the construction of $\left\{q_{t}^{\prime}(x, d y)\right\}$ described in [12], we have the existence of a family $\left\{r_{t}^{\prime}(x, d y)\right\}$ of measures on $K$ satisfying properties (b-2), (b-3(a)) and (b-4) and also

$$
r_{t}^{\prime} f(x)=M_{t}^{P} f(x)+\int_{0}^{t} M_{t-u}^{P}\left[r_{u}^{\prime} f\right](x) d u \text { for all } f \in \mathscr{B}(K), x \in K .
$$

Define $r_{t}^{K}(x, d y) \equiv r_{t}^{\prime}\left(x^{\prime}, d y^{\prime}\right) \times p_{2 t}^{\prime \prime}\left(x^{\prime \prime}, d y^{\prime \prime}\right)$. We will show that

$$
r_{t}^{K} f(x)=M_{t}^{K} f(x)+\int_{0}^{t} M_{t-u}^{K}\left[r_{u}^{R} f\right](x) d u
$$

for all $f \in \mathscr{B}(B), x \in B$.

If $f=\chi_{E}$, where $E=E^{\prime} \times E^{\prime \prime}, E^{\prime} \subset K, E^{\prime \prime} \subset K^{\perp}$, then

$$
\begin{aligned}
M_{t}^{P}\left(x^{\prime}, E^{\prime}\right) \cdot p_{2 t}^{\prime \prime}\left(x^{\prime \prime}, E^{\prime \prime}\right)+ & \int_{0}^{t}\left\{\int_{K} r_{u}^{\prime}\left(y^{\prime}, E^{\prime}\right) \cdot M_{t-u}^{P}\left(x^{\prime}, d y^{\prime}\right)\right\} \\
& \cdot\left\{\int_{\Sigma^{+}} p_{2 u}^{\prime \prime}\left(y^{\prime \prime}, E^{\prime \prime}\right) \cdot p_{2(t-u)}^{\prime \prime}\left(x^{\prime \prime}, d y^{\prime \prime}\right)\right\} d u \\
= & \left\{M_{t}^{P}\left(x^{\prime}, E^{\prime}\right)+\int_{0}^{t} \int_{K} r_{u}^{\prime}\left(y^{\prime}, E^{\prime}\right) \cdot M_{t-u}^{P}\left(x^{\prime}, d y^{\prime}\right) d u\right\} \cdot p_{2 t}^{\prime \prime}\left(x^{\prime \prime}, E^{\prime \prime}\right) \\
= & r_{t}^{\prime}\left(x^{\prime}, E^{\prime}\right) \cdot p_{2 t}^{\prime \prime}\left(x^{\prime \prime}, E^{\prime \prime}\right) \\
= & r_{t}^{K} \chi_{E}(x) .
\end{aligned}
$$


Since the set of all $f \in \mathscr{B}(B)$ which satisfies (7) is closed under finite linear combinations and under bounded monotone limits, it follows as in the proof of Proposition 1 that this set is exactly $\mathscr{B}(B)$. Moreover

$$
\int_{B}\left|r_{t}^{K}\right|(x, d y) \leqq \int_{K}\left|r_{t}^{P}\right|(x, d y),
$$

and since $\left\{r_{t}^{P}(x, d y)\right\}$ satisfies (b-1(a)), we conclude that $\left\{r_{t}^{K}(x, d y)\right\}$ satisfies (b-1(a)). Thus, by Lemma 2.1, $\left\{r_{t}^{K}(x, d y)\right\}$ coincides with the family of measures constructed via the technique of the proof of Proposition 3 of [12] during the construction of the fundamental solution of (5).

To complete the proof of the proposition, we need only establish that $q_{t}^{K}$ satisfies

$$
q_{t}^{K} f(x)=\hat{m}_{t}^{K} f(x)+\int_{0}^{t} \hat{m}_{t-u}^{K}\left[r_{u}^{K} f\right](x) d u
$$

for all $f \in \mathscr{B}(B), x \in B$. If $E$ is a Borel set in $B$ of the form $E^{\prime} \times E^{\prime \prime}$ with $E^{\prime} \subset K$ and $E^{\prime \prime} \subset K^{\perp}$, then

$$
\begin{aligned}
\hat{m}_{t}^{K} \chi_{E}(x)+ & \int_{0}^{t} \hat{m}_{t-u}^{K}\left[\left(r_{u}^{\prime} \times p_{2 u}^{\prime \prime}\right) \chi_{E}\right](x) d u \\
= & \hat{m}_{t}^{P}\left(x^{\prime}, E^{\prime}\right) \cdot p_{2 t}^{\prime \prime}\left(x^{\prime \prime}, E^{\prime \prime}\right)+\int_{0}^{t} \int_{B} r_{u}^{\prime}\left(y^{\prime}, E^{\prime}\right) \cdot p_{2 u}^{\prime \prime}\left(y^{\prime \prime}, E^{\prime \prime}\right) \cdot \hat{m}_{t-u}^{K}(x, d y) \cdot d u \\
= & \hat{m}_{t}^{P}\left(x^{\prime}, E^{\prime}\right) \cdot p_{2 t}^{\prime \prime}\left(x^{\prime \prime}, E^{\prime \prime}\right) \\
& +\int_{0}^{t}\left\{\int_{K} r_{u}^{\prime}\left(y^{\prime}, E^{\prime}\right) \cdot \hat{m}_{t-u}^{P}\left(x^{\prime}, d y^{\prime}\right)\right\} \cdot\left\{\int_{K^{\perp}} p_{2 u}^{\prime \prime}\left(y^{\prime \prime}, E^{\prime \prime}\right) \cdot p_{2(t-u)}^{\prime \prime}\left(x^{\prime \prime}, d y^{\prime \prime}\right)\right\} d u \\
= & \hat{m}_{t}^{P}\left(x^{\prime}, E^{\prime}\right) \cdot p_{2 t}^{\prime \prime}\left(x^{\prime \prime}, E^{\prime \prime}\right) \\
& +\left\{\int_{0}^{t} \int_{K} r_{u}^{\prime}\left(y^{\prime}, E^{\prime}\right) \cdot \hat{m}_{t}^{P}\left(x^{\prime}, d y^{\prime}\right) \cdot d u\right\} \cdot p_{2 t}^{\prime \prime}\left(x^{\prime \prime}, E^{\prime \prime}\right) \\
= & q_{t}^{\prime} \chi_{E^{\prime}}\left(x^{\prime}\right) \cdot p_{2 t}^{\prime \prime} \chi_{E^{\prime \prime}}\left(x^{\prime \prime}\right) \\
= & q_{t}^{K} \chi_{E}(x) .
\end{aligned}
$$

Since the set of all $f \in \mathscr{B}(B)$ which satisfies (8) is closed under finite linear combinations and under bounded monotone limits, it again follows that this set is exactly $\mathscr{B}(B)$. This concludes the proof of Proposition 2.

IV. Convergence of $\left\{\hat{m}_{t}^{K}(x, d y)\right\}$. In the work that follows we will use $c$ to represent a general constant whose dependence may only be on the coefficient operators $A(\cdot)$ and on the relationship of the space $B$ to the space $H$. That is, $c$ will always be independent of $t$ for any $t>0$, independent of any space variables $x, y$, etc., and independent of $P$. All estimates and all formulas will be valid for the case $P=I$ with the obvious modifications.

LEMMA 3.1. Let $\omega$ be a finite positive measure on a space $W$, and $\left\{f_{n}\right\}$ be a sequence of real-valued functions on $W$ which converge almost everywhere (a.e.) to $f$. If $f_{n}$ and f belong to $L^{1+\lambda}(\omega)$ for some $\lambda>0$, with $\left\|f_{n}\right\|_{1+\lambda}$ uniformly bounded, then $f_{n} \rightarrow f\left(L^{1}\right)$. 
Proof. Define

$$
\begin{array}{rlrl}
g_{n}(x) & \equiv \frac{f_{n}(x)-f(x)}{\left|f_{n}(x)\right|+|f(x)|} & & \text { if }\left|f_{n}(x)\right|+|f(x)| \neq 0 \\
& \equiv 0 & & \text { otherwise, } \\
\int_{W}\left|f_{n}-f\right| d \omega & =\int_{W}\left|g_{n}\right|\left(\left|f_{n}\right|+|f|\right) d \omega \leqq\left\|g_{n}\right\|_{\tau} \cdot\left\|\left|f_{n}\right|+|f|\right\|_{1+\lambda}
\end{array}
$$

where $(\tau)^{-1}+(1+\lambda)^{-1}=1$. Since $\left|g_{n}(x)\right| \leqq 1$ for all $x \in W$ and for all $n$, and since $\omega$ is a finite measure, $g_{n} \in L^{\tau} \cdot g_{n} \rightarrow 0$ a.e., and so, by Lebesgue's Dominated Convergence Theorem, $\left\|g_{n}\right\|_{\tau} \rightarrow 0 .\left\|\left|f_{n}\right|+|f|\right\|_{1+\lambda} \leqq\left\|f_{n}\right\|_{1+\lambda}+\|f\|_{1+\lambda} \leqq c$ (independent of $n$ ). Thus $\int_{W}\left|f_{n}-f\right| d \omega \rightarrow 0$.

REMARK. Rather than assume that $f_{n} \rightarrow f$ a.e., it suffices to assume that $g_{n} \rightarrow 0$ in measure (defining $g_{n}$ as in the above proof). For since $\left|g_{n}\right|^{\tau} \leqq 1$, it is a standard measure-theoretic result that again we have $\left\|g_{n}\right\|_{\tau} \rightarrow 0$ for each $1 \leqq \tau<\infty$.

Proposition 3. As $P$ converges to the identity operator on $B, \hat{m}_{t}^{K}(x, d y) \rightarrow$ $\hat{m}_{t}(x, d y)$ in variation, for each $x \in B, t>0$.

Proof. We must show that

$$
\begin{aligned}
(i) \equiv \int_{B} \mid\left[\operatorname{det} A^{K}(y)\right]^{-1 / 2} \exp \left[-\left(C^{K}(x)(x-y), x-y\right) / 4 t\right] \\
-[\operatorname{det} A(y)]^{-1 / 2} \exp [-(C(x)(x-y), x-y) / 4 t] \mid p_{2 t}(d y)
\end{aligned}
$$

converges to zero as $P \rightarrow I$.

$$
\begin{aligned}
& \text { (i) } \leqq \int_{B}\left[\operatorname{det} A^{K}(y)\right]^{-1 / 2} \mid \exp \left[-\left(C^{K}(x)(x-y), x-y\right) / 4 t\right] \\
& -\exp [-(C(x)(x-y), x-y) / 4 t] \mid p_{2 t}(x, d y) \\
& +\int_{B}\left|\left[\operatorname{det} A^{K}(y)\right]^{-1 / 2}-[\operatorname{det} A(y)]^{-1 / 2}\right| \cdot \exp [(C(x)(x-y), x-y) / 4 t] \cdot p_{2 t}(x, d y) \\
& \equiv \text { (ii) }+ \text { (iii), say. }
\end{aligned}
$$

Treating (iii) first, we recall that $A^{K}(y)=I-P B(P y) P . P B(P y) P$ is uniformly (in $P$ and $y$ ) bounded in trace norm. $A^{K}(y)$ is uniformly (in $P$ and $y$ ) bounded away from zero in $L(H, H)$ norm. (Note that $A^{K}(y) \geqq \varepsilon_{0} I$ for all $P$ and $y$, where $\varepsilon_{0}$ is defined in (a-2).) Applying Lemma 4.1 of Gross [6] and noting the Remark on p. 98 of [12], we find that $\left\{\operatorname{det} A^{x}(y)\right\}$ is uniformly bounded both above and away from zero, and

$$
\left|\left[\operatorname{det} A^{R}(y)\right]-[\operatorname{det} A(y)]\right| \leqq c\|P B(P y) P-B(y)\|_{\mathrm{tr}} \text {. }
$$


(If $T \in L(H, H)$, then $\|T\|_{\mathrm{tr}} \equiv \operatorname{trace}\left[\left(T^{*} T\right)^{1 / 2}\right]$.) Since $x^{-1 / 2}$ is Lip-1 on subsets of $(0, \infty)$ which are both bounded above and bounded away from zero, we have

$$
\left|\left[\operatorname{det} A^{K}(y)\right]^{-1 / 2}-[\operatorname{det} A(y)]^{-1 / 2}\right| \leqq c\left|\left[\operatorname{det} A^{K}(y)\right]-[\operatorname{det} A(y)]\right| .
$$

Now if we let $\|\cdot\|_{\mathrm{H}-\mathrm{S}}$ denote the Hilbert-Schmidt norm, we have

$$
\begin{aligned}
\|P B(P y) P-B(y)\|_{\mathrm{tr}} & \leqq\|P B(P y) P-B(P y)\|_{\mathrm{tr}}+\|B(P y)-B(y)\|_{\mathrm{tr}} \\
& =\|(P-I) B(P y) P+B(P y)(P-I)\|_{\mathrm{tr}}+\|B(P y)-B(y)\|_{\mathrm{tr}} \\
& \leqq\left\|Q E B_{0}(P y) E P\right\|_{\mathrm{tr}}+\left\|E B_{0}(P y) E Q\right\|_{\mathrm{tr}}+\|B(P y)-B(y)\|_{\mathrm{tr}} \\
& \leqq c\|Q E\|_{\mathrm{H}-\mathrm{s}}\|E\|_{\mathrm{H}-\mathrm{s}}+\|B(P y)-B(y)\|_{\mathrm{tr}}
\end{aligned}
$$

Since $E$ is Hilbert-Schmidt, $\|Q E\|_{\mathrm{H}-\mathrm{S}} \rightarrow 0$ as $P \rightarrow I$. Since $B(\cdot)$ is Lip-1, $\|B(P y)-B(y)\|_{\mathrm{tr}} \leqq c\|P y-y\|_{B} \rightarrow 0$ pointwise in $y$ as $P \rightarrow I$. Thus

$$
\|P B(P y) P-B(y)\|_{\mathrm{tr}} \rightarrow 0
$$

as $P \rightarrow I$, the convergence being pointwise in $y$. Combining (9), (10) and (11), we conclude that

$$
\left|\left[\operatorname{det} A^{K}(y)\right]^{-1 / 2}-[\operatorname{det} A(y)]^{-1 / 2}\right| \rightarrow 0
$$

as $P \rightarrow I$, the convergence being pointwise in $y$. It is shown in $[12, \mathrm{p} .99]$ that

$$
\exp [-(C(x)(x-y), x-y) / 4 t] \in L^{1+\lambda}\left(p_{2 t}(x, \cdot)\right)
$$

for all positive $\lambda$ which are sufficiently close to zero. For such a $\lambda$, the $L^{1+\lambda}$-norm is uniformly bounded with respect to $x$ and $t$. Thus

$$
\text { (iii) } \leqq c\left\{\int_{B}\left|\left[\operatorname{det} A^{K}(y)\right]^{-1 / 2}-\operatorname{det}[A(y)]^{-1 / 2}\right|^{(1+\lambda) / \lambda} \cdot p_{2 t}(x, d y)\right\}^{\lambda /(1+\lambda)}
$$

The preceding integrand is uniformly (in $P$ and $y$ ) bounded above, and converges pointwise in $y$ to zero as $P \rightarrow I$. Thus, by Lebesgue's Dominated Convergence Theorem, (iii) $\rightarrow 0$ as $P \rightarrow I$. We note that the convergence is not necessarily uniform in $x$ nor in $t$.

Turning now to (ii), we make the change of variables $y \rightarrow x+2 \sqrt{ }$ ty and note that the determinant term is uniformly (in $P, x$ and $t$ ) bounded above, obtaining

$$
\text { (ii) } \leqq c \int_{B}\left|\exp \left[-\left(C^{K}(x) y, y\right)\right]-\exp [-(C(x) y, y)]\right| p_{1 / 2}(d y) .
$$

Now

$$
\begin{aligned}
\left\|C^{K}(x)-C(x)\right\|_{\mathrm{tr}} \leqq & \left\|\left[(I-P B(P x) P)^{-1}-(I-B(x))^{-1}\right] B(x)\right\|_{\mathrm{tr}} \\
& +\left\|(I-P B(P x) P)^{-1}[P B(P x) P-B(x)]\right\|_{\mathrm{tr}} \\
\equiv & (\mathrm{iv})+(\mathrm{v}), \text { say. }
\end{aligned}
$$


$(I-P B(P x) P)^{-1}$ is uniformly (in $P$ and $\left.x\right)$ bounded in $L(H, H)$ norm. It now follows from (11) that (v) $\rightarrow 0$ as $P \rightarrow I$, pointwise in $x$. Writing

$$
\begin{aligned}
(I-P B(P x) P)^{-1}- & (I-B(x))^{-1} \\
= & (I-P B(P x) P)^{-1}(I-B(x))(I-B(x))^{-1} \\
& -(I-P B(P x) P)^{-1}(I-P B(P x) P)(I-B(x))^{-1} \\
= & (I-P B(P x) P)^{-1}[(I-B(x))-(I-P B(P x) P)](I-B(x))^{-1} \\
= & (I-P B(P x) P)^{-1}[P B(P x) P-B(x)](I-B(x))^{-1},
\end{aligned}
$$

we find that

$$
\text { (iv) } \begin{aligned}
& \leqq c\|[P B(P x) P-B(x)] C(x)\|_{\mathrm{tr}} \\
& \leqq c\|P B(P x) P-B(x)\|_{\mathrm{tr}}|C(x)|_{L(H, H)} \\
& \leqq c\|P B(P x) P-B(x)\|_{\mathrm{tr}},
\end{aligned}
$$

and the right-hand side of this inequality converges pointwise to zero as $P \rightarrow I$. Thus $\left\|C^{K}(x)-C(x)\right\|_{\mathrm{tr}} \rightarrow 0$ as $P \rightarrow I$, pointwise in $x$. We use [6, Lemma 1.2] to evaluate

$$
\begin{aligned}
\int_{B} \mid\left(C^{K}(x) y, y\right)-(C(x) y, & y) \mid p_{1 / 2}(d y) \\
& \leqq \int_{B}|| C^{K}(x)-\left.\left.C(x)\right|^{1 / 2} y\right|^{2} p_{1 / 2}(d y) \\
& \left.=\frac{1}{2} \text { (Hilbert-Schmidt norm of }\left|C^{K}(x)-C(x)\right|^{1 / 2}\right)^{2} \\
& =\frac{1}{2}\left\|C^{K}(x)-C(x)\right\|_{\mathrm{tr}}^{2} .
\end{aligned}
$$

Thus $\left(C^{K}(x) y, y\right)^{\sim} \rightarrow(C(x) y, y)^{\sim}$ in mean $\left(p_{1 / 2}\right)$. Since we are in a finite measure space we also have convergence in probability $\left(p_{1 / 2}\right)$. Now for any two real numbers $a$ and $b$,

$$
\begin{aligned}
\left|\frac{e^{a}-e^{b}}{e^{a}+e^{b}}\right| & =\left|\frac{e^{d}}{e^{a}+e^{b}}\right| \cdot|a-b| \text { for some } d \text { between } a \text { and } b \\
& \leqq|a-b| .
\end{aligned}
$$

Therefore if $\left|\left(e^{a}-e^{b}\right) /\left(e^{a}+e^{b}\right)\right|>\varepsilon$, then $|a-b|>\varepsilon$. Consequently,

$p_{1 / 2}\left\{\left|\frac{\exp \left[-\left(C^{K}(x) y, y\right)\right]-\exp [-(C(x) y, y)]}{\exp \left[-\left(C^{K}(x) y, y\right)\right]+\exp [-(C(x) y, y)]}\right|^{\sim}>\varepsilon\right\}$

$$
\leqq p_{1 / 2}\left\{\left|\left(C^{K}(x) y, y\right)^{\sim}-(C(x) y, y)^{\sim}\right|>\varepsilon\right\},
$$

showing that

$$
\left|\frac{\exp \left[-\left(C^{K}(x) y, y\right)\right]-\exp [-(C(x) y, y)]}{\exp \left[-\left(C^{K}(x) y, y\right)\right]+\exp [-(C(x) y, y)]}\right|^{\sim}
$$

converges to zero in probability for each $x \in B$. Since

$$
\left\|\exp \left[-\left(C^{K}(x) y, y\right)\right]\right\|_{1+\lambda}=\operatorname{det}\left[\left(I+(1+\lambda) C^{K}(x)\right)^{-1 / 2}\right]
$$


for $\lambda$ sufficiently small and positive, the calculation on p. 99 of [12] shows that $\left\{\left\|\exp \left[-\left(C^{K}(x) y, y\right)\right]\right\|_{1+\lambda}\right\}$ is uniformly bounded with respect to $P$ and $x$. By Lemma 3.1 and the remark which follows it, we conclude that (ii) $\rightarrow 0$ as $P \rightarrow I$, the convergence being independent of $t$ but not necessarily of $x$. This concludes the proof of Proposition 3.

V. Estimates on the coefficients. We again note that unless specified otherwise all estimates will be valid for the case $P=I(K=H)$ with the obvious modifications.

(c-1) There exists a symmetric Hilbert-Schmidt class operator $F$ on $H$ and a family of operators $C_{0}^{K}(x) \in L(H, H)$ such that for all $x \in B, C^{K}(x)=F C_{0}^{K}(x) F$ and $\left|C_{0}^{K}(x)\right| \leqq 1 . F$ is independent of $P$ (i.e. of $K$ ).

We follow the proof of c-2) of [12]. Since the operator $E$ of (a-3) commutes with $P$ it is easy to see that such an $F$ exists for each $P$. However, to see that $F$ can be chosen independently of $P$, we will go through the necessary calculations.

If $P^{\prime}$ is chosen from our special family of projections, and if $Q^{\prime} \equiv I-P^{\prime}$, then

$$
\begin{aligned}
I-P B(P x) P & =I-\left(P^{\prime}+Q^{\prime}\right) P B(P x) P\left(P^{\prime}+Q^{\prime}\right) \\
& =\left[I-P^{\prime} P B(P x) P P^{\prime}\right]-\left[P^{\prime} P B(P x) P Q^{\prime}+Q^{\prime} P B(P x) P\right] .
\end{aligned}
$$

Since $\left|P^{\prime} P B(P x) P Q^{\prime}+Q^{\prime} P B(P x) P\right| \leqq c\left|E Q^{\prime}\right|$, we may choose $P^{\prime}$ to satisfy

$$
\left|P^{\prime} P B(P x) P Q^{\prime}+Q^{\prime} P B(P x) P\right| \leqq\left(1-\varepsilon_{0}\right) \varepsilon_{0} .
$$

The $\varepsilon_{0}$ used above is the $\varepsilon_{0}$ of hypothesis (a-2). Factoring out $\left[I-P^{\prime} P B(P x) P P^{\prime}\right]$, we obtain

$$
I-P B(P x) P=\left[I-P^{\prime} P B(P x) P P^{\prime}\right]\left[I-D^{K}(x)\right]
$$

where

$$
\begin{aligned}
D^{K}(x) & \equiv\left[I-P^{\prime} P B(P x) P P^{\prime}\right]^{-1} E\left[P^{\prime} P B_{0}(P x) P Q^{\prime}+Q^{\prime} P B_{0}(P x) P\right] E . \\
\left|D^{K}(x)\right| \leqq 1-\varepsilon_{0} . & \text { Also, for } y_{1}, y_{2} \in H \\
& \left|\left(D^{K}(x) y_{1}, y_{2}\right)\right| \leqq 2\left|E y_{1}\right| \cdot\left|E\left[I-P^{\prime} P B(P x) P P^{\prime}\right]^{-1} y_{2}\right|
\end{aligned}
$$

Now

$$
\begin{aligned}
\mid E\left[I-P^{\prime} P B\right. & \left.(P x) P P^{\prime}\right]\left.^{-1} y_{2}\right|^{2} \\
& =\left|E\left[I-P^{\prime} P B(P x) P P^{\prime}\right]^{-1} P^{\prime} y_{2}+E\left[I-P^{\prime} P B(P x) P P^{\prime}\right]^{-1} Q^{\prime} y_{2}\right|^{2} \\
& \leqq|E|^{2} \varepsilon_{0}^{-2}\left|P^{\prime} y_{2}\right|^{2}+\left|E Q^{\prime} y_{2}\right|^{2} \\
& \leqq\left|E_{1} y_{2}\right|^{2}
\end{aligned}
$$

where $E_{1} \equiv 2\left[|E| \varepsilon_{0}^{-1} P^{\prime}+E Q^{\prime}\right]$. Since $E_{1}$ is symmetric and of Hilbert-Schmidt class 
and $2\left|E y_{1}\right| \leqq\left|E_{1} y_{1}\right|$, it follows from [6, Lemma 4.2] that we may write $D^{y}(x)=$ $E_{1} D_{0}^{K}(x) E_{1}$, where $\left|D_{0}^{K}\right| \leqq 1$. Expanding,

$$
\begin{aligned}
{\left[I-D^{K}(x)\right]^{-1} } & =I+\lim _{n \rightarrow \infty} \sum_{i=1}^{n}\left[D^{K}(x)\right]^{i} \\
& =I+E_{1}\left\{D_{0}^{K}(x)+\lim _{n \rightarrow \infty} \sum_{i=0}^{n} D_{0}^{K}(x) E_{1}\left[D^{K}(x)\right]^{i} E_{1} D_{0}^{K}(x)\right\} E_{1} \\
& \equiv I+E_{1} D_{1}^{K}(x) E_{1}, \quad \text { say, }
\end{aligned}
$$

where $\left|D_{1}^{K}(x)\right| \leqq 1+\left|E_{1}\right|^{2} \varepsilon_{0}^{-1}$. Thus

$$
\begin{aligned}
\left|\left(C^{K}(x) y_{1}, y_{2}\right)\right|= & \left|\left(\left[I-D^{K}(x)\right]^{-1}\left[I-P^{\prime} P B(P x) P P^{\prime}\right]^{-1} P B(P x) P y_{1}, y_{2}\right)\right| \\
\leqq & \left|\left(B_{0}(P x) P E y_{1}, P E\left[I-P^{\prime} P B(P x) P P^{\prime}\right]^{-1} y_{2}\right)\right| \\
& +\left|\left(D_{1}^{K}(x) E_{1}\left[I-P^{\prime} P B(P x) P P^{\prime}\right]^{-1} P E B_{0}(P x) P E y_{1}, E_{1} y_{2}\right)\right| \\
\leqq & \left|E y_{1}\right| \cdot\left|E_{1} y_{2}\right|+\left[1+\left|E_{1}\right|^{2} \varepsilon_{0}^{-1}\right] \cdot\left|E_{1}\right| \cdot \varepsilon_{0}^{-1} \cdot|E| \cdot\left|E y_{1}\right| \cdot\left|E_{1} y_{2}\right| \\
\leqq & \left|a E y_{1}\right| \cdot\left|E_{1} y_{2}\right|
\end{aligned}
$$

where $a \equiv 1+\left[1+\left|E_{1}\right|^{2} \varepsilon_{0}^{-1}\right] \cdot\left|E_{1}\right| \cdot \varepsilon_{0}^{-1} \cdot|E|$. (c-1) now follows on applying Lemma 4.2 of [6] together with the argument following b-5) of [12].

(c-2) There exist families of operators $C_{1}^{K}(x) \in L(B \rightarrow L(H, H))$ and $C_{2}^{K}(x) \in$ $L(B \rightarrow L(B \rightarrow L(H, H)))$ such that for all $x, z, z_{1}, z_{2} \in B$ we have $D C^{K}(x) z=$ $F C_{1}^{K}(x) z F$ and $D^{2} C^{K}(x) z_{1} z_{2}=F C_{2}^{K}(x) z_{1} z_{2} F$ with $\left\|C_{1}^{K}(x)\right\| \leqq 1$ and $\left\|C_{2}^{K}(x)\right\| \leqq 1$.

$D C^{K}(x) z$ is given for all $x, z \in B$ by the formula

$$
\begin{aligned}
D C^{K}(x) z= & {[I-P B(P x) P]^{-1} P[D B(P x) z] P[I-P B(P x) P]^{-1} P B(P x) P } \\
& +[I-P B(P x) P]^{-1} P[D B(P x) z] P \\
= & {[I-P B(P x) P]^{-1} P[D B(P x) z] P\left[(I-P B(P x) P]^{-1} .\right.}
\end{aligned}
$$

We note for future reference that $D C^{K}(x) z$ depends only on $P z$, since $B(P x)$ depends only on $P x$ and so $D B(P x) z=0$ for all $z \in K^{\perp}$. Moreover, $D C^{K}(x) z$ acts as the zero operator on $K^{\perp}$, and $K$ is invariant under $D C^{K}(x) z$. For each $y_{1}, y_{2} \in H$ we have

$$
\left|\left(D C^{K}(x) z y_{1}, y_{2}\right)\right| \leqq\left|D B_{0}(P x) z\right| \cdot\left|E[I-P B(P x) P]^{-1} y_{1}\right| \cdot\left|E[I-P B(P x) P]^{-1} y_{2}\right| .
$$

From (14) and (16) we obtain

$$
\begin{aligned}
E[I-P B(P x) P]^{-1} & =E\left[I-D^{K}(x)\right]^{-1}\left[I-P^{\prime} P B(P x) P P^{\prime}\right]^{-1} \\
& =E\left[I-P^{\prime} P B(P x) P P^{\prime}\right]^{-1}+E E_{1} D_{1}^{K}(x) E_{1}\left[I-P^{\prime} P B(P x) P P^{\prime}\right]^{-1} .
\end{aligned}
$$

It now follows from (15) and from a similar estimate for $\left|E_{1}\left[I-P^{\prime} P B(P x) P P^{\prime}\right]^{-1} y\right|$ that there exists a symmetric Hilbert-Schmidt class operator $F_{1}$ on $H$ such that

$$
\left|\left(D C^{K}(x) z y_{1}, y_{2}\right)\right| \leqq\left|F_{1} y_{1}\right| \cdot\left|F_{1} y_{2}\right| \cdot\|z\| .
$$

Without loss of generality we may assume that $F_{1}=F$. Lemma 4.2 of [6] now gives the desired result for $D C^{K}(x) z$.

The calculations for $D^{2} C^{K}(x)$ follow without difficulty from the above estimates. 
(c-3) As $P \rightarrow I,\left|[I-P B(P x) P]^{-1}-[I-B(x)]^{-1}\right| \rightarrow 0$ pointwise for all $x \in B$.

From (13) we have

$$
[I-P B(P x) P]^{-1}-[I-B(x)]^{-1}=[I-P B(P x) P]^{-1}[P B(P x) P-B(x)][I-B(x)]^{-1} .
$$

Since $\left|[I-P B(P x) P]^{-1}\right|$ is uniformly (in $x$ and $P$ ) bounded, we have

$$
\begin{aligned}
\left|[I-P B(P x) P]^{-1}-[I-B(x)]^{-1}\right| & \leqq c|P B(P x) P-B(x)| \\
& \leqq c\|P B(P x) P-B(x)\|_{\mathrm{tr}}
\end{aligned}
$$

and the right-hand side of the previous inequality converges to zero as $P \rightarrow I$, by (11).

(c-4) As $P \rightarrow I,|P[D B(P x) z] P-D B(x) z| \rightarrow 0$ pointwise for $x \in B$ and uniformly for $z$ varying over a bounded set in $B$.

Let $Q \equiv I-P$. Then

$$
\begin{aligned}
&|P[D B(P x) z] P-D B(x) z| \\
& \leqq|P[D B(P x) z] P-D B(P x) z|+|D B(P x) z-D B(x) z| \\
&=|[P-I][D B(P x) z] P+[D B(P x) z][P-I]|+\left|E\left[D B_{0}(P x) z-D B_{0}(x) z\right] E\right| \\
& \leqq\left|Q E\left[D B_{0}(P x) z\right] E P\right|+\left|E\left[D B_{0}(P x) z\right] E Q\right|+c\left\|D B_{0}(P x)-D B_{0}(x)\right\| \cdot\|z\| \\
& \leqq c\|z\| \cdot|E Q|+c\|P x-x\| \cdot\|z\|
\end{aligned}
$$

by (a-5), and the right-hand side of the above inequality converges to zero as $P \rightarrow I$, the convergence being pointwise in $x$ and uniform on bounded sets of $\|z\|$.

(c-5) As $P \rightarrow I,\left|D C^{K}(x) z-D C(x) z\right| \rightarrow 0$ pointwise for $x \in B$ and uniformly for $z$ varying over a bounded set in $B$.

Noting that

$$
\begin{aligned}
&\left|D C^{K}(x) z-D C(x) z\right|=\mid[I-P B(P x) P]^{-1} P[D B(P x) z] P[I-P B(P x) P]^{-1} \\
&-[I-B(x)]^{-1} D B(x) z[I-B(x)]^{-1} \mid,
\end{aligned}
$$

the result follows immediately from (c-3) and (c-4).

(c-6) As $P \rightarrow I,\left|P\left[D^{2} B(P x) z_{1} z_{2}\right] P-D^{2} B(x) z_{1} z_{2}\right| \rightarrow 0$ pointwise for $x \in B$ and uniformly for $z_{1}$ and $z_{2}$ varying over bounded sets in $B$.

Setting $Q \equiv I-P$, we have

$$
\begin{aligned}
\left|P\left[D^{2} B(P x) z_{1} z_{2}\right] P-D^{2} B(x) z_{1} z_{2}\right| & \\
\leqq & \left|[P-I]\left[D^{2} B(P x) z_{1} z_{2}\right] P+\left[D^{2} B(P x) z_{1} z_{2}\right][P-I]\right| \\
& +\left|D^{2} B(P x) z_{1} z_{2}-D^{2} B(x) z_{1} z_{2}\right| \\
\leqq & \left|Q E\left[D^{2} B_{0}(P x) z_{1} z_{2}\right] E P\right|+\left|E\left[D^{2} B_{0}(P x) z_{1} z_{2}\right] E Q\right| \\
& +\left|E\left[D^{2} B_{0}(P x)-D^{2} B_{0}(x)\right] z_{1} z_{2} E\right| \\
\leqq & c|Q E| \cdot\left\|z_{1}\right\| \cdot\left\|z_{2}\right\|+c\left\|D^{2} B_{0}(P x)-D^{2} B_{0}(x)\right\| \cdot\left\|z_{1}\right\| \cdot\left\|z_{2}\right\| \\
\leqq & c\left\|z_{1}\right\| \cdot\left\|z_{2}\right\| \cdot[|Q E|+\|P x-x\|] .
\end{aligned}
$$

$|Q E| \rightarrow 0$ as $P \rightarrow I$, and $\|P x-x\| \rightarrow 0$, pointwise in $x$, as $P \rightarrow I$. 
(c-7) As $P \rightarrow I,\left|D^{2} C^{K}(x) z_{1} z_{2}-D^{2} C(x) z_{1} z_{2}\right| \rightarrow 0$ pointwise for $x \in B$ and uniformly for $z_{1}$ and $z_{2}$ varying over bounded sets in $B$.

We have the formula

$$
\begin{aligned}
D^{2} C^{K}(x) z_{1} z_{2}- & D^{2} C(x) z_{1} z_{2}=[I-P B(P x) P]^{-1} \\
\cdot\{ & P\left[D B(P x) z_{2}\right] P[I-P B(P x) P]^{-1} P\left[D B(P x) z_{1}\right] P \\
+ & P\left[D^{2} B(P x) z_{1} z_{2}\right] P \\
+ & \left.P\left[D B(P x) z_{1}\right] P[I-P B(P x) P]^{-1} P\left[D B(P x) z_{2}\right] P\right\}[I-P B(P x) P]^{-1} \\
& -[I-B(x)]^{-1}\left\{\left[D B(x) z_{2}\right][I-B(x)]^{-1}\left[D B(x) z_{1}\right]+\left[D^{2} B(x) z_{1} z_{2}\right]\right. \\
& \left.+\left[D B(x) z_{1}\right][I-B(x)]^{-1}\left[D B(x) z_{2}\right]\right\}[I-B(x)]^{-1} .
\end{aligned}
$$

(c-7) now follows on using estimates (c-3), (c-4) and (c-6).

The next set of estimates that we shall make will be integral estimates. The measurability of most of the functions which we will use follows from either [12, Lemma 1] or else from the following lemma.

LEMMA 4.1. Let $M$ be a normed linear space with norm $|\cdot|_{M}$ and let $H^{(N)} \equiv H \times$ $H \times \cdots \times H(N$ times $)$. Let $f\left(y_{1}, \ldots, y_{N}\right): H^{(N)} \rightarrow M$ be linear in each $y_{i}$. If $\left|f\left(y_{1}, \ldots, y_{N}\right)\right|_{M} \leqq c\left|y_{1}\right|_{1} \cdot\left|y_{2}\right|_{2} \cdots \cdot\left|y_{N}\right|_{N}$ where $|\cdot|_{i}$ is a measurable seminorm on $H$ $(i=1, \ldots, N)$, then $G(y) \equiv|f(y, \ldots, y)|_{M}$ is u.c.n. 0 in $H_{m}$.

The proof of this lemma is similar to that of Lemma 1 of [12], once we observe that

$$
|G(y)-G(z)| \leqq|f(y, y, \ldots, y)-f(z, z, \ldots, z)|_{M} .
$$

(c-8) As $P \rightarrow I, \int_{B}\left|\left(\left[C^{K^{\prime}}(x)-C^{\prime}(x)\right] y y, y\right)\right| p_{2 t}(d y) \rightarrow 0$ pointwise for $x \in B$ and $t>0$.

Set $Q \equiv I-P$ and write

$$
\begin{aligned}
\left(C^{\prime}(x) y y, y\right)= & \left(\left[C^{\prime}(x)\right](P y)(P y), P y\right)+\left(\left[C^{\prime}(x)\right](P y)(P y), Q y\right) \\
& +\left(\left[C^{\prime}(x)\right](P y)(Q y), y\right)+\left(\left[C^{\prime}(x)\right](Q y) y, y\right),
\end{aligned}
$$

valid for each $y \in H$. Noting that $\left(C^{K^{\prime}}(x) y y, y\right)^{\sim}=\left(\left[C^{K^{\prime}}(x)\right](P y)(P y), P y\right)$ for a.e. $y \in B$, we have

$$
\begin{aligned}
& \int_{B}\left|\left(\left[C^{R^{\prime}}(x)-C^{\prime}(x)\right] y y, y\right)\right| p_{2 t}(d y) \\
& \leqq \int_{P H}\left|\left(\left[C^{K^{\prime}}(x)-C^{\prime}(x)\right](P y)(P y), P y\right)\right| \nu_{2 t}(d y) \\
&+\int_{B}(\|P y\| \cdot|F P y| \cdot|F Q y|+\|P y\| \cdot|F Q y| \cdot|F y|) p_{2 t}(d y) \\
&+\int_{B}\|Q y\| \cdot|F y|^{2} \cdot p_{2 t}(d y) \\
& \equiv(\mathrm{i})+(\mathrm{ii})+(\mathrm{iii}), \text { say. }
\end{aligned}
$$


The integrand of (iii) is bounded by $c\|y\| \cdot|F y|^{2 \sim}$ for a.e. $y \in B$ and moreover it converges to zero a.e. on $B$ as $P \rightarrow I$. Thus (iii) $\rightarrow 0$ as $P \rightarrow I$ by Dominated Convergence.

$$
\text { (ii) } \begin{aligned}
& \leqq c \int_{B}\|y\| \cdot|F y| \cdot|F Q y| \cdot p_{2 t}(d y) \\
& \leqq c\left\{\int_{B}(\|y\| \cdot|F y|)^{2} p_{2 t}(d y)\right\}^{1 / 2}\left\{\int_{B}|F Q y|^{2} p_{2 t}(d y)\right\}^{1 / 2} \\
& \leqq c\left\{\operatorname{trace}[(I-P) F]^{2}\right\}^{1 / 2} \\
& \rightarrow 0 \text { as } P \rightarrow I .
\end{aligned}
$$

Let $S_{n}$ denote the sphere in $H$ with center at the origin and with radius $n$. We have

$$
\begin{aligned}
\int_{P H / P S_{n}} \mid\left(\left[C^{K^{\prime}}(x)-C^{\prime}(x)\right](P y)(P y)\right. & , P y) \mid \nu_{2 t}(d y) \\
& \leqq c \int_{P H / P S_{n}}\|P y\| \cdot|F P y|^{2} \cdot \nu_{2 t}(d y) \\
& \leqq c\left\{\int_{P H}\|P y\|^{2} \nu_{2 t}(d y)\right\}^{1 / 2}\left\{\int_{P H / P S_{n}}|F P y|^{4} \nu_{2 t}(d y)\right\}^{1 / 2} \\
& =c\left\{\int_{B}\|P y\|^{2} p_{2 t}(d y)\right\}^{1 / 2}\left\{\int_{P H / P S_{n}}|F P y|^{4} \nu_{2 t}(d y)\right\}^{1 / 2} \\
& \leqq c\left\{\int_{B}\|y\|^{2} p_{2 t}(d y)\right\}^{1 / 2}\left\{\int_{P H / P S_{n}}|F P y|^{4} \nu_{2 t}(d y)\right\}^{1 / 2} \\
& \leqq c\left\{\int_{P H / P S_{n}}|F P y|^{4} \nu_{2 t}(d y)\right\}^{1 / 2} .
\end{aligned}
$$

We claim that the right-hand side of the previous inequality converges to zero as $n \rightarrow \infty$, the convergence being independent of $P$. Since $P H$ is invariant under $F$ (all $P$ 's commute with $F$ ), we can choose a basis $\left\{e_{i}: i=1, \ldots, m\right\}$ for $P H$ consisting of eigenvectors of $F$. Let $\lambda_{i} \equiv F e_{i}$ and $y \equiv \sum_{i=1}^{m} y_{i} e_{i}$. Then

$$
\begin{aligned}
\int_{P H / P S_{n}}|F P y|^{4} \nu_{2 t}(d y) & \\
= & \frac{1}{(4 \pi t)^{m / 2}} \int_{P H / P S_{n}}\left(\sum_{i=1}^{m} \lambda_{i}^{2} y_{1}^{2}\right)^{2} \exp \left(-\sum_{i=1}^{m} y_{i}^{2} / 4 t\right) d y_{1} \cdots d y_{m} \\
& =(4 \pi t)^{-m / 2} \int_{P H / P S_{n}}\left(\sum_{i, j=1}^{m} \lambda_{i}^{2} \lambda_{j}^{2} y_{i}^{2} y_{j}^{2}\right) \exp \left(-\sum_{i=1}^{m} y_{i}^{2} / 4 t\right) d y_{1} \cdots d y_{m} .
\end{aligned}
$$

Given $\varepsilon>0$, for each $i=1, \ldots, m$,

$$
\begin{aligned}
(4 \pi t)^{-m / 2} \int_{P H / P S_{n}} \lambda_{i}^{4} y_{i}^{4} \exp \left(-\sum_{j=1}^{m} y_{j}^{2} / 4 t\right) d y_{1} \cdots d y_{m} & \\
& \leqq(4 \pi t)^{-1 / 2} \lambda_{i}^{4} \int_{|y| \geqq n} y^{4} \exp \left(-y^{2} / 4 t\right) d y
\end{aligned}
$$


and $\int_{|y| \geqq n} y^{2} \exp \left(-y^{2} / 4 t\right) d y$ can be made $<\varepsilon(4 \pi t)^{1 / 2}$ for $n$ sufficiently large. Similarly,

$$
\begin{gathered}
(4 \pi t)^{-m / 2} \int_{P H / P S_{n}} \lambda_{i}^{2} \lambda_{j}^{2} y_{i}^{2} y_{j}^{2} \exp \left(-\sum_{k=1}^{m} y_{i}^{2} / 4 t\right) d y_{1} \cdots d y_{m} \\
\leqq \lambda_{i}^{2} \lambda_{j}^{2}\left((4 \pi t)^{-1 / 2} \int_{|y| \geqq n} y^{2} \exp \left(-y^{2} / 4 t\right) d y\right)^{2}
\end{gathered}
$$

where $\int_{|y| \geqq n} y^{2} \exp \left(-y^{2} / 4 t\right) d y$ can be made $<(4 \pi t \varepsilon)^{1 / 2}$ for $n$ sufficiently large. Thus

$$
\begin{aligned}
\int_{P H / P S_{n}}|F P y|^{4} \nu_{2 t}(d y) & \leqq \varepsilon \sum_{i, j=1}^{m} \lambda_{i}^{2} \lambda_{j}^{2} \\
& =\varepsilon\left(\sum_{i=1}^{m} \lambda_{i}^{2}\right)^{2} \leqq \varepsilon\left(\operatorname{trace} F^{2}\right)^{2},
\end{aligned}
$$

for all $n$ sufficiently large and for all $P$.

Now for any fixed $n$ we may make use of estimate (c-5) to choose $P_{0}$ so that $P \supseteq P_{0}$ implies that

for all $y \in S_{n}$. Then

$$
\left|\left(\left[C^{K^{\prime}}(x)-C^{\prime}(x)\right] y y, y\right)\right|<\varepsilon
$$

$$
\int_{P S_{n}}\left|\left(\left[C^{K^{\prime}}(x)-C^{\prime}(x)\right](P y)(P y), P y\right)\right| \nu_{2 t}(d y)<\varepsilon
$$

and so (i) $\rightarrow 0$ as $P \rightarrow I$, pointwise for $x \in B$. This concludes the proof of (c-8).

(c-9) As $P \rightarrow I$,

$$
\int_{B}\left\|\left(\left[C^{K^{\prime \prime}}(x)-C^{\prime \prime}(x)\right](\cdot)(\cdot) y, y\right)\right\|_{\mathrm{tr}} p_{2 t}(d y) \rightarrow 0
$$

pointwise for $x \in B$ and $t>0$.

Set $Q \equiv I-P$ and write

$$
\begin{aligned}
\left(C^{\prime \prime}(x)(\cdot)(\cdot) y, y\right)= & \left(C^{\prime \prime}(x)(\cdot)(\cdot)(P y), P y\right)+\left(C^{\prime \prime}(x)(\cdot)(\cdot)(P y), Q y\right) \\
& +\left(C^{\prime \prime}(x)(\cdot)(\cdot)(Q y), y\right),
\end{aligned}
$$

valid for each $y \in H$. Since

$$
\left\|\left(C^{K^{\prime \prime}}(x)(\cdot)(\cdot) y, y\right)\right\|_{\mathrm{tr}}=\left\|\left(C^{K^{\prime \prime}}(x)(\cdot)(\cdot)(P y), P y\right)\right\|_{\mathrm{tr}}
$$

for a.e. $y \in B$, we have

$$
\begin{aligned}
& \int_{B}\left\|\left(\left[C^{K^{\prime \prime}}(x)-C^{\prime \prime}(x)\right](\cdot)(\cdot) y, y\right)\right\|_{\mathrm{tr}} p_{2 t}(d y) \\
& \leqq \int_{P H}\left\|\left(\left[C^{K^{\prime \prime}}(x)-C^{\prime \prime}(x)\right](\cdot)(\cdot)(P y), P y\right)\right\|_{\mathrm{tr}} \nu_{2 t}(d y) \\
&+\int_{B}(|F P y| \cdot|F Q y|+|F Q y| \cdot|F y|) p_{2 t}(d y) \\
& \equiv(\mathrm{i})+(\mathrm{ii}), \text { say. }
\end{aligned}
$$




$$
\text { (ii) } \begin{aligned}
& \leqq c \int_{B}|F y| \cdot|F Q y| \cdot p_{2 t}(d y) \\
& \leqq c\left\{\int_{B}|F y|^{2} p_{2 t}(d y)\right\}^{1 / 2}\left\{\int_{B}|F Q y|^{2} p_{2 t}(d y)\right\}^{1 / 2} \\
& \leqq c\left\{\operatorname{trace}((I-P) F)^{2}\right\}^{1 / 2} \\
& \rightarrow 0 \text { as } P \rightarrow I .
\end{aligned}
$$

Let $S_{n}$ denote the sphere in $H$ with center at the origin and with radius $n$. We have

$$
\begin{aligned}
\int_{P S_{n}} \|\left(\left[C^{K^{\prime \prime}}(x)-C^{\prime \prime}(x)\right](\cdot)(\cdot)(P y)\right. & , P y) \|_{t \mathrm{tr}} \nu_{2 t}(d y) \\
& \leqq c \int_{P S_{n}}\left\|D^{2}\left(C^{K}(x)-C(x)\right)\right\| \cdot n^{2} \cdot \nu_{2 t}(d y) \\
& \leqq c n^{2} \sup _{\left\|z_{1}\right\|,\left\|z_{2}\right\| \leqq 1}\left|D^{2}\left(C^{K}(x)-C(x)\right) z_{1} z_{2}\right| \\
& \rightarrow 0 \quad \text { as } P \rightarrow I, \quad \text { by estimate (c-7). }
\end{aligned}
$$

Now

$$
\int_{P H / P S_{n}}\left\|\left(\left[C^{K^{\prime \prime}}(x)-C^{\prime \prime}(x)\right](\cdot)(\cdot)(P y), P y\right)\right\|_{t r} \nu_{2 t}(d y) \leqq c \int_{P H / P S_{n}}|F P y|^{2} \nu_{2 t}(d y) .
$$

As in the proof of (c-8) we can show that the right-hand side of the previous inequality converges to zero as $n \rightarrow \infty$, the convergence being independent of $P$. It follows that (i) $\rightarrow 0$ as $P \rightarrow I$.

$$
\text { (c-10) As } P \rightarrow I
$$

$$
\int_{B}\left\|\left(C^{K^{\prime}}(x)(\cdot) y, y\right) \otimes\left(C^{K^{\prime}}(x)(\cdot) y, y\right)-\left(C^{\prime}(x)(\cdot) y, y\right) \otimes\left(C^{\prime}(x)(\cdot) y, y\right)\right\|_{\mathrm{tr}} p_{2 t}(d y)
$$

converges to zero, pointwise for $x \in B$ and $t>0$.

For $h \in H$ we will write $h^{2}$ for $h \otimes h .\left(C^{K^{\prime}}(x)(\cdot) y, y\right)$ is, for each $x \in B$ and $y \in H$, a bounded linear functional on $H$. Thus it may be identified with an element $h \in H$ with $|h| \leqq c|F y|^{2}$. Noting that the constant is independent of the choice of $P$ and that $\|h \otimes h\|_{\mathrm{tr}}=|h|^{2}$ (see [14]), we obtain

$$
\left\|\left(C^{K^{\prime}}(x)(\cdot) y, y\right)^{2}-\left(C^{\prime}(x)(\cdot) y, y\right)^{2}\right\|_{\operatorname{tr}} \leqq c|F y|^{4},
$$

valid for each $y \in H$. This ensures that the integral in (c-10) exists, and also that the integrand arises from a function on $H$ which is u.c.n. 0 in $H_{m}$.

Set $Q \equiv I-P$, and for $y \in H$ write

$$
\left(C^{\prime}(x)(\cdot) y, y\right)=\left(C^{\prime}(x)(\cdot)(P y), P y\right)+\left(C^{\prime}(x)(\cdot)(P y), Q y\right)+\left(C^{\prime}(x)(\cdot)(Q y), y\right) .
$$


Noting that $\left(C^{K^{\prime}}(x)(\cdot) y, y\right)=\left(C^{K^{\prime}}(x)(\cdot)(P y), P y\right)$ for each $y \in H$, we have

$$
\begin{aligned}
& \int_{B}\left\|\left(C^{K^{\prime}}(x)(\cdot) y, y\right)^{2}-\left(C^{\prime}(x)(\cdot) y, y\right)^{2}\right\|_{\operatorname{tr}} p_{2 t}(d y) \\
& \leqq \int_{P H}\left\|\left(C^{K^{\prime}}(x)(\cdot)(P y), P y\right)^{2}-\left(C^{\prime}(x)(\cdot)(P y), P y\right)^{2}\right\|_{\mathrm{tr}} \nu_{2 t}(d y) \\
& +\int_{B} \|\left(C^{\prime}(x)(\cdot)(P y), P y\right) \otimes\left[\left(C^{\prime}(x)(\cdot)(P y), Q y\right)+\left(C^{\prime}(x)(\cdot)(Q y), y\right)\right] \\
& +\left[\left(C^{\prime}(x)(\cdot)(P y), Q y\right)+\left(C^{\prime}(x)(\cdot)(Q y), y\right)\right]^{2} \\
& +\left[\left(C^{\prime}(x)(\cdot)(P y), Q y\right)+\left(C^{\prime}(x)(\cdot)(Q y), y\right)\right] \\
& \quad \otimes\left(C^{\prime}(x)(\cdot)(P y), P y\right) \|_{\mathrm{tr}} p_{2 t}(d y)
\end{aligned}
$$

Using the fact that $\left\|h_{1} \otimes h_{2}\right\|_{\mathrm{tr}}=\left|h_{1}\right| \cdot\left|h_{2}\right|$ for each $h_{1}, h_{2} \in H$, we obtain

$$
\begin{aligned}
& \text { (ii) } \leqq c \int_{B}\left\{|F P y|^{2}[|F P y| \cdot|F Q y|+|F Q y| \cdot|F y|]\right. \\
& +[|F P y| \cdot|F Q y|+|F Q y| \cdot|F y|]^{2} \\
& \left.+[|F P y| \cdot|F Q y|+|F Q y| \cdot|F y|] \cdot|F P y|^{2}\right\} p_{2 t}(d y) \\
& \leqq c \int_{B}\left[|F y|^{3}|F Q y|+|F y|^{2}|F Q y|^{2}\right] p_{2 t}(d y) \\
& \leqq c \int_{B}|F y|^{3}|F Q y| p_{2 t}(d y) \\
& \leqq c\left\{\int_{B}|F y|{ }^{6} p_{2 t}(d y)\right\}^{1 / 2}\left\{\int_{B}|F Q y|^{2} p_{2 t}(d y)\right\}^{1 / 2} \\
& \leqq c\left\{\text { trace }((I-P) F)^{2}\right\}^{1 / 2} \\
& \rightarrow 0 \text { as } P \rightarrow I \text {. }
\end{aligned}
$$

For $h_{1}, h_{2} \in H$ we have

$$
\left\|h_{1}^{2}-h_{2}^{2}\right\|_{\text {tr }}=\left\|\left(h_{1}-h_{2}\right) \otimes h_{1}+h_{2} \otimes\left(h_{1}-h_{2}\right)\right\|_{\text {tr }} \leqq\left(\left|h_{1}\right|+\left|h_{2}\right|\right) \cdot\left|h_{1}-h_{2}\right| .
$$

Thus

$$
\text { (i) } \left.\leqq c \int_{P H}|F P y|^{2} \cdot \sup _{h \in H:|h|=1}\left\{\left[C^{K^{\prime}}(x)-C^{\prime}(x)\right] h(P y), P y\right)\right\} \cdot \nu_{2 t}(d y) .
$$

Using (c-5) and proceeding in a manner similar to that used for (i) of (c-8) or (c-9), we find that (i) $\rightarrow 0$ as $P \rightarrow I$, the convergence being pointwise for $x \in B$.

(c-11) As $P \rightarrow I$,

$$
\int_{B}\left\|\left(C^{K^{\prime}}(x)(\cdot)(\cdot), y\right)-\left(C^{\prime}(x)(\cdot)(\cdot), y\right)\right\|_{\operatorname{tr}} p_{2 t}(d y) \rightarrow 0,
$$

pointwise for $x \in B$ and $t>0$. 
We recall that for each $y \in H$ the operators $T^{K} \equiv\left(C^{K^{\prime}}(\cdot)(\cdot), y\right)$ are defined for all $h_{1}, h_{2} \in H$ by

$$
\left(T^{K} h_{1}, h_{2}\right)=\frac{1}{2}\left[\left(C^{K^{\prime}}(x) h_{1} h_{2}, y\right)+\left(C^{K^{\prime}}(x) h_{2} h_{1}, y\right)\right]
$$

Each $T^{K}$ is symmetric and of Hilbert-Schmidt class. The symmetry is obvious from the definition; to demonstrate the Hilbert-Schmidt property we will show that $T^{K}$ is a sum of two Hilbert-Schmidt class operators. If $\left\{e_{i}\right\}$ is any orthonormal basis for $H$, then

$$
\begin{aligned}
\sum_{i=1}^{\infty} \sup _{h \in H ;|h|=1}\left|\left(C^{K^{\prime}}(x) h e_{i}, y\right)\right|^{2} & =\sum_{i=1}^{\infty} \sup _{h \in H:|h|=1}\left|\left(F C_{1}^{K}(x) h F e_{i}, y\right)\right|^{2} \\
& \leqq c|F y|^{2} \cdot \sum_{i=1}^{\infty}\left|F e_{i}\right|^{2}<\infty
\end{aligned}
$$

and

$$
\begin{aligned}
\sum_{i=1}^{\infty} \sup _{h \in H:|h|=1}\left|\left(C^{K^{\prime}}(x) e_{i} h, y\right)\right|^{2} \\
=\sum_{i=1}^{\infty} \sup _{h \in H:|h|=1}\left|\left([I-P B(P x) P]^{-1} P D B(P x) e_{i} P[I-B(P x) P]^{-1} h, y\right)\right|^{2} \\
\quad \leqq \sum_{i=1}^{\infty}\left|E[I-P B(P x) P]^{-1} y\right|^{2} \cdot\left|D B_{0}(P x) e_{i}\right|^{2} \cdot \sup _{h \in H ;|h|=1}\left|E[I-P B(P x) P]^{-1} h\right|^{2} \\
\quad \leqq c_{y} \cdot \sum_{i=1}^{\infty}\left|D B_{0}(P x) e_{i}\right|^{2}
\end{aligned}
$$

where $c_{y}$ is a constant depending only on $y$. The last sum is finite, by hypothesis (a-6).

To show now that each $T^{K}$ is of trace class, it now suffices to show that $\sum_{i=1}^{\infty}\left|\left(T^{K} e_{i}, e_{i}\right)\right|<\infty$ for each $\left\{e_{i}\right\}$. We have

$$
\begin{aligned}
\left|\left(T^{K}(x) e_{i}, e_{i}\right)\right| & =\left|\left([I-P B(P x) P]^{-1} E P\left[D B_{0}(P x) e_{i}\right] P E[I-P B(P x) P]^{-1} e_{i}, y\right)\right| \\
& \leqq\left|D B_{0}(P x) e_{i}\right| \cdot\left|E[I-P B(P x) P]^{-1} e_{i}\right| \cdot\left|E[I-P B(P x) P]^{-1} y\right|
\end{aligned}
$$

where

$$
\begin{aligned}
\left|E[I-P B(P x) P]^{-1} y\right| & =\left|E\left[I-P^{\prime} P B(P x) P P^{\prime}\right]\left[I-D^{x}(x)\right] y\right| \quad \text { by (14) } \\
& \leqq\left|E\left[I-P^{\prime} P B(P x) P P^{\prime}\right] y\right|+c\left|D^{x}(x) y\right| \\
& \leqq\left|E_{1} y\right|+c|E y|
\end{aligned}
$$

by (15) and the definition of $D^{X}(x) . E_{1}$ and $c$ are independent of $P, x$ and $y$, and both $E_{1}$ and $E$ are of Hilbert-Schmidt class. Thus there exists a Hilbert-Schmidt 
class operator $E_{2} \in L(H, H)$ such that for all $y \in H$ we have $\left|E[I-P B(P x) P]^{-1} y\right| \leqq$ $\left|E_{2} y\right|$. Then

$$
\begin{aligned}
\sum_{i=1}^{\infty}\left|\left(T^{K}(x) e_{i}, e_{i}\right)\right| & \leqq \sum_{i=1}^{\infty}\left|D B_{0}(P x) e_{i}\right| \cdot\left|E_{2} e_{i}\right| \cdot\left|E_{2} y\right| \\
& \leqq\left|E_{2} y\right| \cdot\left\{\sum_{i=1}^{\infty}\left|D B_{0}(P x) e_{i}\right|^{2}\right\}^{1 / 2} \cdot\left\{\sum_{i=1}^{\infty}\left|E_{2} e_{i}\right|^{2}\right\}^{1 / 2} \\
& \leqq c\left|E_{2} y\right|
\end{aligned}
$$

by (a-6). It follows that $\left\|T^{K}(x)\right\|_{\mathrm{tr}} \leqq c\left|E_{2} y\right|$. This ensures that the integrand in (c-11) exists and that it arises from a function which is u.c.n. 0 in $H_{m}$.

We now estimate the integrand by

$$
\begin{aligned}
\left\|\left(C^{K^{\prime}}(x)(\cdot)(\cdot), y\right)-\left(C^{\prime}(x)(\cdot)(\cdot), y\right)\right\|_{\mathrm{tr}} \leqq & \left\|\left(C^{K^{\prime}}(x)(\cdot)(\cdot), P y\right)-\left(C^{\prime}(x)(\cdot)(\cdot), P y\right)\right\|_{\mathrm{tr}} \\
& +\left\|\left(C^{\prime}(x)(\cdot)(\cdot), Q y\right)\right\|_{\mathrm{tr}} \\
\equiv & (\mathrm{i})+(\mathrm{ii}), \quad \text { say. }
\end{aligned}
$$

If we can show that (i) $\rightarrow 0$ as $P \rightarrow I$, pointwise for each $x \in B$ and uniformly for $y$ in a bounded set in $H$, then the techniques employed in proving (c-8)-(c-10) will give us (c-11). Note that for any orthonormal basis $\left\{e_{i}\right\}$ of $H$

$$
\begin{aligned}
& \sum_{i=1}^{\infty}\left|\left(C^{K^{\prime}}(x) e_{i} e_{i}, P y\right)-\left(C^{\prime}(x) e_{i} e_{i}, P y\right)\right| \\
& \leqq|P y| \cdot \sum_{i=1}^{\infty} \mid\left[[I-P B(P x) P]^{-1} P\left[D B(P x) e_{i}\right] P[I-P B(P x) P]^{-1}\right. \\
& \left.-[I-B(x)]^{-1} D B(x) e_{i}[I-B(x)]^{-1}\right] e_{i} \mid .
\end{aligned}
$$

It suffices to show that the latter sum converges (for each $x \in B$ ) to zero as $P \rightarrow I$, and that the convergence is independent of the choice of basis $\left\{e_{i}\right\}$. This sum may be written as

$$
\begin{aligned}
& \sum_{i=1}^{\infty} \mid\left\{[I-P B(P x) P]^{-1}-[I-B(x)]^{-1}\right\} P\left[D B(P x) e_{i}\right] P[I-P B(P x) P]^{-1} e_{i} \\
&+[I-B(x)]^{-1}\left\{P\left[D B(P x) e_{i}\right] P-D B(x) e_{i}\right\}[I-P B(P x) P]^{-1} e_{i} \\
&+[I-B(x)]^{-1} D B(x) e_{i}\left\{[I-P B(P x) P]^{-1}-[I-B(x)]^{-1}\right\} e_{i} \mid \\
& \leqq c\left|[I-P B(P x) P]^{-1}-[I-B(x)]^{-1}\right| \cdot \sum_{i=1}^{\infty}\left|D B_{0}(P x) e_{i}\right| \cdot\left|E[I-P B(P x) P]^{-1} e_{i}\right| \\
&+c \sum_{i=1}^{\infty}\left|\left[D B_{0}(P x)-D B_{0}(x)\right] e_{i}\right| \cdot\left|E[I-P B(P x) P]^{-1} e_{i}\right| \\
&+c \sum_{i=1}^{\infty}\left|D B_{0}(x) e_{i}\right| \cdot\left|E\left\{[I-P B(P x) P]^{-1}-[I-B(x)]^{-1}\right\} e_{i}\right| \\
& \equiv(\text { iii) }+ \text { (iv) }+(\mathrm{v}), \text { say. }
\end{aligned}
$$


(c-3), (a-6) and the fact that $E$ is Hilbert-Schmidt give us the result that (iii) $\rightarrow 0$ as $P \rightarrow I$, independent of the choice of $\left\{e_{i}\right\}$; (a-9) gives us the same result for (iv); and (v) follows from (a-6) and the calculation

$$
\begin{aligned}
\left\{\sum_{i=1}^{\infty}\left|E\left\{[I-P B(P x) P]^{-1}-[I-B(x)]^{-1}\right\} e_{i}\right|^{2}\right\}^{1 / 2} & \\
& \leqq\left|[I-P B(P x) P]^{-1}-[I-B(x)]^{-1}\right|_{L(H, H)}\|E\|_{\mathrm{H}-\mathrm{s}} \\
& \rightarrow 0 \text { as } P \rightarrow I, \quad \text { by (c-3). }
\end{aligned}
$$

VI. Convergence of $\left\{M_{t}^{K}(x, d y)\right\},\left\{r_{t}^{K}(x, d y)\right\}$ and $\left\{q_{t}^{K}(x, d y)\right\}$.

Proposition 4. As $P \rightarrow I, M_{t}^{K}(x, d y) \rightarrow M_{t}(x, d y)$ in variation norm, for each $x \in B, t>0$.

Proof. It follows from (4) that we may write

$$
\begin{aligned}
M_{t}^{K}(x, d y)=\left[\operatorname{det} A^{K}(x+y)\right]^{-1 / 2} \cdot\left\{\operatorname{trace}\left[A^{K}(x)\right] \cdot[\right. & (-4 t)^{-1}\left(C^{K^{\prime \prime}}(x)(\cdot)(\cdot) y, y\right) \\
& +t^{-1}\left(C^{K^{\prime}}(x)(\cdot)(\cdot), y\right) \\
& \left.+\left(16 t^{2}\right)^{-1}\left(C^{K^{\prime}}(x)(\cdot) y, y\right)^{2}\right] \\
& \left.+(-4 t)^{-1}\left(C^{K^{\prime}}(x) y y, y\right)\right\}^{\sim} \\
& \cdot \exp \left[-\left(C^{K}(x) y, y\right) / 4 t\right]^{\sim} \cdot p_{2 t}(d y) .
\end{aligned}
$$

This formula, with the obvious modifications, also holds for $P=I$. To simplify the notation in this proof, we will fix $x$ and $t$ and write

$$
\begin{aligned}
& a(y) \equiv[\operatorname{det} A(x+y)]^{-1 / 2}, \quad b \equiv A(x), \\
& d(y) \equiv(-4 t)^{-1}\left(C^{\prime \prime}(x)(\cdot)(\cdot) y, y\right)+t^{-1}\left(C^{\prime}(x)(\cdot)(\cdot), y\right)+\left(16 t^{2}\right)^{-1}\left(C^{\prime}(x)(\cdot) y, y\right)^{2}, \\
& e(y) \equiv(-4 t)^{-1}\left(C^{\prime}(x) y y, y\right), \quad f(y) \equiv \exp [-(C(x) y, y) / 4 t],
\end{aligned}
$$

and $a^{K}, \ldots, f^{K}$ for the above functions with $A$ and $C$ replaced by $A^{K}$ and $C^{K}$ respectively. We must show that

$$
\int_{B}\left|a^{K}\left[\operatorname{trace}\left(b^{K} d^{K}\right)+e^{K}\right] f^{K}-a[\operatorname{trace}(b d)+e] f\right| p_{2 t}(d y) \rightarrow 0
$$

as $P \rightarrow I$. The integrand may be written as

$$
\begin{aligned}
& \mid\left[a^{K}-a\right][\operatorname{trace}(b d)+e] f+a^{K}\left\{\operatorname{trace}\left[\left(b^{K}-b\right) d\right]+\operatorname{trace}\left[b^{K}\left(d^{K}-d\right)\right]\right\} f^{K} \\
& +a^{K}\left(e^{K}-e\right) f^{K}+\left.a^{K}[\operatorname{trace}(b d)+e]\left[f^{K}-f\right]\right|^{\sim} \\
& \leqq\left\{\left|a^{K}-a\right| \cdot|\operatorname{trace}(b d)+e| \cdot|f|+\left|a^{K}\right| \cdot\left|b^{K}-b\right|_{L(H, H)} \cdot\|d\|_{\mathrm{tr}} \cdot\left|f^{K}\right|\right. \\
& +\left|a^{K}\right| \cdot\left|b^{K}\right|_{L(H, H)} \cdot\left\|d^{K}-d\right\|_{\mathrm{tr}} \cdot\left|f^{K}\right|+\left|a^{K}\right| \cdot\left|e^{K}-e\right| \cdot\left|f^{K}\right| \\
& \left.+\left|a^{K}\right| \cdot \mid \text { trace }(b d)+e|\cdot| f^{K}-f \mid\right\}^{\sim} \text {. }
\end{aligned}
$$

From [12, pp. 98-99] we find that for any $\lambda>0$

$$
\int_{B}\left|f^{K}\right|^{1+\lambda} p_{2 t}(d y)=\operatorname{det}\left[\left(I+(1+\lambda) C^{K}(x)\right)^{-1 / 2}\right] .
$$


Moreover there exists an $\varepsilon_{1}>0$, independent of $P$ and $x$, such that for $\lambda<\varepsilon_{1}$ we have $\left[I+(1+\lambda) C^{K}(x)\right]>0$ and $\left|[I+(1+\lambda) C(x)]^{-1}\right|$ is uniformly bounded for all $x \in B$. Thus, by Lemma 4.1 of [6], for any fixed $\lambda<\varepsilon_{1}, \int_{B}\left|f^{K}\right|^{1+\lambda} p_{2 t}(d y)$ is uniformly bounded for $x \in B$, for all $P$, and for all $t>0$. For $\lambda<\varepsilon_{1}$ we claim that $\left|f^{K}-f\right| \rightarrow 0$ in $L^{1+\lambda}\left(p_{2 t}\right)$ as $P \rightarrow I$. This is equivalent to saying that $\left|g^{K}-g\right| \rightarrow 0$ in $L^{1+\lambda}\left(p_{1 / 2}\right)$, where $g^{K} \equiv \exp \left[-\left(C^{K}(x) y, y\right)\right]$ and $g \equiv g^{H}$. To prove the latter, we write

$$
\begin{aligned}
& \int_{B}\left|g^{K}-g\right|^{1+\lambda} p_{1 / 2}(d y) \\
&=\int_{B}\left|\frac{g^{K}-g}{\left|g^{K}\right|+|g|}\right|^{1+\lambda}\left(\left|g^{K}\right|+|g|\right)^{1+\lambda} p_{1 / 2}(d y) \\
& \leqq\left\{\int_{B}\left|\frac{g^{K}-g}{\left|g^{K}\right|+|g|}\right|^{(1+\lambda) \tau} p_{1 / 2}(d y)\right\}^{1 / \tau}\left\{\int_{B}\left(\left|g^{K}\right|+|g|\right)^{(1+\lambda)(1+\rho)} p_{1 / 2}(d y)\right\}^{1 /(1+\rho)}
\end{aligned}
$$

where $(\tau)^{-1}+(1+\rho)^{-1}=1$. $\left(\left|g^{K}\right|+|g|\right) \in L^{(1+\lambda)(1+\rho)}$ if $(1+\lambda)(1+\rho)<1+\varepsilon_{1}$. This condition is satisfied iff $\lambda+\rho(1+\lambda)<\varepsilon_{1}$ or, equivalently, iff $\rho<\left(\varepsilon_{1}-\lambda\right)(1+\lambda)^{-1}$. Since $\left(\varepsilon_{1}-\lambda\right)>0$, we can always find such a $\rho$. The result now follows once we observe that $\left|\left(g^{K}-g\right) /\left(\left|g^{K}\right|+|g|\right)\right| \sim$ is bounded by 1 a.e. on $B$ and converges to zero in probability by a previous calculation for the proof of Proposition 3 .

$a^{K} \rightarrow a$ pointwise for all $y \in B$. Since $\left|a^{K}\right| \leqq c$, it follows that $\left|a^{K}-a\right| \rightarrow 0$ in $L^{q}\left(p_{2 t}\right)$ for all $1 \leqq q<\infty$ and for all $t>0$. $b^{K} \rightarrow b$ in $L(H, H)$ norm, $\left|b^{K}\right|_{L(H, H)}$ is uniformly bounded with respect to $P$, and thus, since $b^{K}$ is independent of $y \in B$ we have $\left|b^{K}-b\right|_{L(H, H)} \rightarrow 0$ in $L^{q}\left(p_{2 t}\right)$ for all $1 \leqq q<\infty$ and for all $t>0$.

$\left\|d^{K}-d\right\|_{\mathrm{tr}} \rightarrow 0$ in $L^{1}\left(p_{2 t}\right)$ by (c-9), (c-10) and (c-11), and $\left|e^{K}-e\right| \rightarrow 0$ in $L^{1}\left(p_{2 t}\right)$ by (c-8). The $L^{1}$ convergence of these functions to zero implies their $L^{q}$ convergence to zero for any $1 \leqq q<\infty$. For if we have a sequence of functions $\left\{g^{K}\right\}$, say, such that $\left|g^{K}\right|$ is dominated a.e. for each $K$ by a function $h$ which is in $L^{q}\left(p_{2 t}\right)$ for each $1 \leqq q<\infty$, then

$$
\int_{B}\left|g^{K}-g\right| p_{2 t}(d y) \rightarrow 0
$$

is equivalent to $\left|g^{K}-g\right| \rightarrow 0$ in probability $\left(p_{2 t}\right)$ which, in turn, is equivalent to $\left|g^{K}-g\right|^{q} \rightarrow 0$ in probability $\left(p_{2 t}\right)$ and this is equivalent to

$$
\int_{B}\left|g^{K}-g\right|^{q} p_{2 t}(d y) \rightarrow 0 .
$$

It remains to check that $d^{K}$ and $e^{K}$ are dominated by the correct type of function. This follows immediately from

$$
\left\|d^{K}\right\|_{\mathrm{tr}} \leqq(4 t)^{-1}|F y|^{2}+c t^{-1}\left|E_{2} y\right|+\left(16 t^{2}\right)^{-1}|F y|^{4},
$$

and

$$
\left|e^{K}\right| \leqq(4 t)^{-1}\|y\| \cdot|F y|^{2} .
$$

Proposition 4 follows from the above estimates and several applications of Hölder's inequality. 
Proposition 5. As $P \rightarrow I, r_{t}^{R}(x, d y) \rightarrow r_{t}(x, d y)$ in variation norm, for each $x \in B$, $t>0$.

Proof. We may write

$$
r_{t}^{K}(x, d y)=\sum_{n=1}^{\infty} r_{t, n}^{K}(x, d y)
$$

where $r_{t, 1}^{K}(x, d y) \equiv M_{t}^{K}(x, d y)$ and $r_{t, n}^{K}(x, d y)$ is defined for $n \geqq 2$ by

$$
r_{t, n}^{K}(x, d y) \equiv \int_{0}^{t} \int_{B} \int_{B} f(z) \cdot r_{u, n-1}^{K}(y, d z) \cdot M_{t-u}^{K}(x, d y) \cdot d u
$$

for all $f \in \mathscr{B}(B), x \in B$ and $t>0$. From the estimates of [12] it is easy to see that there exists a constant $Q$, independent of $P, x$ and $t$, such that

$$
\int_{B}\left|M_{t}^{K}\right|(x, d y) \leqq Q t^{-1 / 2}
$$

and

$$
\int_{B}\left|r_{t, n}^{K}\right|(x, d y) \leqq Q^{n} \pi^{n / 2} t^{n / 2-1} / \Gamma(n / 2)
$$

Thus, given $\varepsilon>0$, there exists an $N(\varepsilon$ ), independent of $P$ and $x$ (but dependent on $t$ ) such that

$$
\int_{B}\left|r_{t}^{K}-r_{t}\right|(x, d y) \leqq \sum_{n=1}^{N(\varepsilon)} \int_{B}\left|r_{t, n}^{K}-r_{t, n}\right|(x, d y)+\varepsilon
$$

Now $r_{t, 1}^{K}(x, d y)=M_{t}^{K}(x, d y)$, so $r_{t, 1}^{K}(x, d y) \rightarrow r_{t, 1}(x, d y)$ in variation by Proposition 4. If we assume that $r_{t, k}^{K}(x, d y) \rightarrow r_{t, k}(x, d y)$ in variation for all $k \leqq n-1, x \in B$ and $t>0$, then

$$
\begin{aligned}
\int_{B} \mid r_{t, n}^{K} & -r_{t, n} \mid(x, d y) \\
& \leqq \int_{0 ;(u)}^{t} \int_{B ;(y)} \int_{B ;(z)}\left|r_{u, n-1}^{K}(y, d z) \cdot M_{t-u}^{K}(x, d y)-r_{u, n-1}(y, d z) \cdot M_{t-u}(x, d y)\right| d u \\
& \leqq \int_{0 ;(u)}^{t} \int_{B ;(y)} \int_{B ;(z)}\left|r_{u, n-1}^{K}-r_{u, n-1}\right|(y, d z) \cdot\left|M_{t-u}\right|(x, d y) \cdot d u \\
& \quad+\int_{0 ;(u)}^{t} \int_{B ;(y)} \int_{B ;(z)}\left|r_{u, n-1}^{K}\right|(y, d z) \cdot\left|M_{t-u}^{K}-M_{t-u}\right|(x, d y) \cdot d u \\
& \equiv \text { (i)+(ii), say. }
\end{aligned}
$$

We consider (i). $\int_{B}\left|r_{u, n-1}^{K}-r_{u, n-1}\right|(y, d z) \rightarrow 0$ as $P \rightarrow I$ for each $u>0$ and $y \in B$. Moreover, by (17)

$$
\int_{B}\left|r_{u, n-1}^{K}-r_{u, n-1}\right|(y, d z) \leqq 2 \frac{Q^{n-1} \pi^{(n-1) / 2}}{\Gamma((n-1) / 2)} u^{n / 2-3 / 2}
$$


for all $P$. Since

$$
\int_{0}^{t} \int_{B} u^{n / 2-3 / 2} \cdot\left|M_{t-u}\right|(x, d y) \cdot d u \leqq Q \int_{0}^{t} u^{n / 2-3 / 2} \cdot(t-u)^{-1 / 2} \cdot d u
$$

and the integral on the right-hand side exists, we conclude by Dominated Convergence that (i) $\rightarrow 0$ as $P \rightarrow I$.

We consider (ii).

$$
\text { (ii) } \leqq \frac{Q^{n-1} \pi^{(n-1) / 2}}{\Gamma((n-1) / 2)} \int_{0}^{t} \int_{B}\left|M_{t-u}^{K}-M_{t-u}\right|(x, d y) \cdot u^{n / 2-3 / 2} \cdot d u \text {. }
$$

Now $\int_{B}\left|M_{t-u}^{K}-M_{t-u}\right|(x, d y) \rightarrow 0$ for each $u>0$ and moreover

$$
\int_{B}\left|M_{t-u}^{K}-M_{t-u}\right|(x, d y)
$$

is dominated (for all $P$ and for all $x \in B$ ) by $2 Q(t-u)^{-1 / 2}$, which is integrable from 0 to $t$ with respect to $u^{(n-3) / 2} d u$. Thus (ii) $\rightarrow 0$ as $P \rightarrow I$, and so $r_{t, k}^{K}(x, d y) \rightarrow$ $r_{t, k}(x, d y)$ for each $k=1,2, \ldots$ The proposition now follows from (18).

Proposition 6. As $P \rightarrow I, q_{t}^{K}(x, d y) \rightarrow q_{t}(x, d y)$ in variation norm, for each $x \in B$, $t>0$.

\section{Proof.}

$$
\begin{aligned}
& \int_{B}\left|q_{t}^{K}-q_{t}\right|(x, d y) \\
& \qquad \int_{B}\left|\hat{m}_{t}^{K}-\hat{m}_{t}\right|(x, d y) \\
& \quad+\int_{0:(u)}^{t} \int_{B:(y)} \int_{B:(z)}\left|r_{u}^{K}(y, d z) \cdot \hat{m}_{t-u}^{K}(x, d y)-r_{u}(y, d z) \cdot \hat{m}_{t-u}(x, d y)\right| \cdot d u \\
& \equiv(\mathrm{i})+(\mathrm{ii}), \quad \text { say. }
\end{aligned}
$$

Now (i) $\rightarrow 0$ as $P \rightarrow I$ by Proposition 3, and

$$
\text { (ii) } \begin{aligned}
\int_{0:(u)}^{t} \int_{B ;(y)} \int_{B ;(z)} \mid r_{u}^{K}- & r_{u}|(y, d z) \cdot| \hat{m}_{t-u} \mid(x, d y) \cdot d u \\
& \quad+\int_{0:(u)}^{t} \int_{B ;(y)} \int_{B ;(z)}\left|r_{u}^{K}\right|(y, d z) \cdot\left|\hat{m}_{t-u}^{K}-\hat{m}_{t-u}\right|(x, d y) \cdot d u .
\end{aligned}
$$

From [12, Proposition 3], $\int_{B}\left|r_{t}^{K}\right|(x, d y) \leqq c_{t_{0}} t^{-1 / 2}$, where $c_{t_{0}}$ is a constant independent of $t$ and of $x$. $c_{t_{0}}$ can easily be seen to be independent of $P$. Also $\int_{B}\left|\hat{m}_{t}^{K}\right|(x, d y)$ is dominated by a constant, independent of $P, x$ and $t$. By an argument similar to that of Proposition 5 we may now establish that (ii) $\rightarrow 0$ as $P \rightarrow I$.

COROllary 6.1. For each $t>0, x \in B, q_{t}(x, d y)$ is a probability measure on $B$.

This is an immediate consequence of Proposition 6. 


\section{The semigroup property.}

THEOREM 1. For $f \in \mathscr{B}(B), s$ and $t>0, q_{t} q_{s} f(x)=q_{t+s} f(x)$.

Proof. Let $f \in \mathscr{B}(B)$, and for a finite signed Borel measure $\mu$ on $B$ let $\|\mu\|$ denote the total variation of $\mu$. Denoting the measure $q_{t}(x, d y)$ by $q_{t, x}(d y)$ we have

$$
\left|\left(q_{t} q_{s}-q_{t+s}\right) f(x)\right| \leqq\left|q_{t}\left(q_{s}-q_{s}^{K}\right) f(x)\right|+\left|\left(q_{t}-q_{t}^{K}\right) q_{s}^{K} f(x)\right|+\left|\left(q_{t+s}^{K}-q_{t+s}\right) f(x)\right|,
$$

where

$$
\left|q_{t}\left(q_{s}-q_{s}^{K}\right) f(x)\right| \leqq\|f\|_{\infty} \int_{B ;(y)} \int_{B ;(z)}\left|q_{s}-q_{s}^{K}\right|(y, d z) \cdot\left|q_{t}\right|(x, d y) .
$$

Now $\int_{B}\left|q_{s}-q_{s}^{K}\right|(y, d z)$ converges to 0 as $P \rightarrow I$ pointwise for $y \in B$. Moreover this term is dominated by 2. It now follows by Dominated Convergence that $\left|q_{t}\left(q_{s}-q_{s}^{K}\right) f(x)\right| \rightarrow 0$ as $P \rightarrow I$. Considering the two remaining terms, we have

$$
\left|\left(q_{t}-q_{t}^{K}\right) q_{s}^{K} f(x)\right| \leqq\left\|q_{t, x}-q_{t, x}^{K}\right\| \cdot\left\|q_{s}^{K} f\right\|_{\infty} \leqq\left\|q_{t, x}-q_{t, x}^{K}\right\| \cdot\|f\|_{\infty}
$$

and

$$
\left|\left(q_{t+s}^{K}-q_{t+s}\right) f(x)\right| \leqq\left\|q_{t+s, x}^{K}-q_{t+s, x}\right\| \cdot\|f\|_{\infty},
$$

both of which converge to zero as $P \rightarrow I$ by Proposition 6 .

\section{BIBLIOGRAPHY}

1. Z. Ciesielski, On Haar functions and on the Schauder basis of the space $C_{\langle 0,1\rangle}$, Bull. Acad. Polon. Sci. Sér. Sci. Math. Astronom. Phys. 7 (1959), 227-232. MR 24 \#A1599.

2. F. G. Dressel, The fundamental solution of the parabolic equation, Duke Math. J. 7 (1940), 186-203. MR 2, 204.

3. - The fundamental solution of the parabolic equation. II, Duke Math. J. 13 (1946), 61-70. MR 7, 450.

4. E. B. Dynkin, Markov processes, Fizmatgiz, Moscow, 1963; English transl., Vols. I, II, Academic Press, New York and Springer-Verlag, Berlin and New York, 1965. MR 33 \#1886; 1887.

5. A. Friedman, Partial differential equations of parabolic type, Prentice-Hall, Englewood Cliffs, N. J. 1964. MR 31 \#6062.

6. L. Gross, Integration and nonlinear transformations in Hilbert space, Trans. Amer. Math. Soc. 94 (1960), 404-440. MR 22 \#2883.

7. - Measurable functions on Hilbert space, Trans. Amer. Math. Soc. 105 (1962), 372-390. MR $26 \# 5121$.

8. - Abstract Wiener spaces, Proc. Fifth Berkeley Sympos. Math. Statist. and Prob. (Berkey, Calif., 1965/66), vol. II, part I, Univ. of California Press, Berkeley, 1967, pp. 31-42. MR 35 \#3027.

9. - - Potential theory on Hilbert space, J. Functional Analysis 1 (1967), 123-181. MR 37 \#3331.

10. A. M. Il'in, A. S. Kalašnikov and O. A. Oleĭnik, Second-order linear equations of parabolic type, Uspehi Mat. Nauk 17 (1962), no. 3 (105), 3-146= Russian Math. Surveys 17 (1962), no. 3, 1-143. MR 25 \#2328. 
11. S. Itô, The fundamental solution of the parabolic equation in a differentiable manifold, Osaka Math. J. 5 (1953), 75-92. MR 15, 36.

12. M. A. Piech, A fundamental solution of the parabolic equation on Hilbert space, J. Functional Analysis 3 (1969), 85-114.

13. - Regularity properties for families of measures on a metric space, Proc. Amer. Math. Soc. 24 (1970), 307-311.

14. R. Schatten, A theory of cross-spaces, Ann. of Math. Studies, no. 26, Princeton Univ. Press, Princeton, N. J., 1950. MR 12, 186.

15. B. Sz.-Nagy, Introduction to real functions and orthogonal expansions, Oxford Univ. Press, New York, 1965. MR 31 \#5938.

State University of New York at Buffalo, AMHerst, NeW YorK 14226 